Pacific Northwest National Laboratory

Operated by Battelle for the

U.S. Department of Energy

\section{Hanford Site Environmental Surveillance Master Sampling Schedule for Calendar Year 2008}

L. E. Bisping

January 2008

Prepared for the U.S. Department of Energy under Contract DE-AC05-76RL01830 


\title{
DISCLAIMER
}

This report was prepared as an account of work sponsored by an agency of the United States Government. Reference herein to any specific commercial product, process, or service by trade name, trademark, manufacturer, or otherwise does not necessarily constitute or imply its endorsement, recommendation, or favoring by the United States Government or any agency thereof, or Battelle Memorial Institute. This report is a summary of major or significant activities occurring at the Hanford Site only, and is not a full disclosure of all details associated with Hanford-related activities, nor a substitute for legally required information subject to reporting requirements regarding releases, violations, etc.

\author{
PACIFIC NORTHWEST NATIONAL LABORATORY \\ operated by \\ BATTELLE \\ for the \\ UNITED STATES DEPARTMENT OF ENERGY \\ under Contract DE-AC05-76RL01830
}

Printed in the United States of America
May be available to DOE and DOE contractors from the
Office of Scientific and Technical Information,
P.O. Box 62, Oak Ridge, TN 37831-0062;
ph: (865) 576-8401
fax: (865) 576-5728
email: reports@adonis.osti.gov

\author{
May be available to the public from the National Technical Information Service, \\ U.S. Department of Commerce, 5285 Port Royal Rd., Springfield, VA 22161 \\ ph: (800) 553-6847 \\ fax: (703) 605-6900 \\ email: orders@ntis.fedworld.gov \\ online ordering: http://www.ntis.gov/ordering.htm
}

This document was printed on recycled paper. 


\title{
Hanford Site Environmental Surveillance Master Sampling Schedule for Calendar Year 2008
}

\author{
L. E. Bisping
}

January 2008

Prepared for

the U.S. Department of Energy

under Contract DE-AC05-76RL01830

Pacific Northwest National Laboratory

Richland, Washington 


\section{Summary}

Environmental surveillance of the Hanford Site and surrounding areas is conducted by the Pacific Northwest National Laboratory (PNNL) ${ }^{\text {a }}$ for the U.S. Department of Energy (DOE). Sampling is conducted to evaluate levels of radioactive and nonradioactive pollutants in the Hanford environs, as required in DOE Order 450.1, Environmental Protection Program ${ }^{\mathrm{b}}$, and DOE Order 5400.5, Radiation Protection of the Public and the Environment ${ }^{c}$. The environmental surveillance sampling design is described in the Hanford Site Environmental Monitoring Plan, United States Department of Energy, Richland Operations Office (DOE/RL-91-50 ${ }^{\mathrm{d}}$ ).

This document contains the calendar year 2008 schedule for the routine collection of samples for the Surface Environmental Surveillance Project (SESP) and Drinking Water Monitoring Project (DWMP). Each section includes sampling locations, sampling frequencies, sample types, and analyses to be performed. In some cases, samples are scheduled on a rotating basis. If a sample will not be collected in 2008, the anticipated year for collection is provided. Maps showing approximate sampling locations are included for media scheduled for collection in 2008.

\section{Surface Environmental Surveillance Project Sampling}

The SESP is a multimedia environmental surveillance effort to measure the concentrations of radionuclides and chemicals in environmental media to demonstrate compliance with applicable environmental quality standards and public exposure limits, and assessing environmental impacts. Project personnel annually collect selected samples of ambient air, surface water, agricultural products, fish, wildlife, and sediments. Soil and vegetation samples are collected approximately every 5 years. Analytical capabilities include the measurement of radionuclides at very low environmental concentrations and, in selected media, nonradiological chemicals including metals, anions, volatile organic compounds, and total organic carbon.

\section{Drinking Water Monitoring Project Sampling}

Fluor Hanford, Inc. is responsible for monitoring the quality of drinking water supplied by DOE to its onsite facilities in accordance with federal and state regulations. PNNL conducts radiological monitoring of onsite drinking water for Fluor Hanford, Inc. concurrent with SESP activities to promote sampling efficiency and consistency, utilize expertise developed over the years, and reduce costs associated with management, sample collection, procedure development, analytical contracting, data management, quality control, and reporting.

\footnotetext{
${ }^{\text {a} P a c i f i c ~ N o r t h w e s t ~ N a t i o n a l ~ L a b o r a t o r y ~ i s ~ o p e r a t e d ~ b y ~ B a t t e l l e ~ f o r ~ t h e ~ U . S . ~ D e p a r t m e n t ~ o f ~ E n e r g y . ~}$ bDOE Order 450.1. 2003. “Environmental Protection Program.” U.S. Department of Energy, Washington, D.C. 'DOE Order 5400.5. 1990. "Radiation Protection of the Public and the Environment.” U.S. Department of Energy, Washington, D.C.

${ }^{\mathrm{d}}$ DOE/RL-91-50, Rev. 3. 2000. Environmental Monitoring Plan, United States Department of Energy Richland Operations Office. U.S. Department of Energy, Richland, Washington.
} 


\section{Data Management}

The Hanford Environmental Information System (HEIS) database is used as a repository for data gathered during environmental surveillance activities at the Hanford Site. For ease in retrieving SESP or drinking water data from the HEIS database (HEIS 1989), the majority of the location names in this document are the location names used in the database.

\section{Schedule Changes}

This schedule is subject to modification during the year in response to changes in site operations, program requirements, and the nature of the observed results. Operational limitations such as weather, mechanical failures, sample availability, and other factors may also impact scheduled sampling. Therefore, this document may not be an accurate record of samples collected during the year.

\section{Multi-Agency Samples}

By joint agreement, some samples are collected by SESP personnel and provided to the Washington State Department of Health (DOH) and the U.S. Food and Drug Administration (FDA). All planned cooperative sampling efforts are indicated in this schedule.

\section{Additional Information}

Questions relating to the content of this document can be directed to T. M. (Ted) Poston, Manager, SESP, (509) 372-6900 or G. W. (Greg) Patton, Manager, DWMP, (509) 371-7071. 


\section{Acronyms and Symbols}

\section{Acronyms}

$\begin{array}{ll}\text { ALE } & \text { Fitzner/Eberhardt Arid Lands Ecology Reserve } \\ \text { DOH } & \text { Washington State Department of Health } \\ \text { DR } & \text { Downriver (from noted location) } \\ \text { FDA } & \text { U.S. Food and Drug Administration } \\ \text { FFTF } & \text { Fast Flux Test Facility } \\ \text { HRM } & \text { Hanford river markers } \\ \text { ICP-MS } & \text { Inductively coupled plasma mass spectrometry } \\ \text { NSQAN } & \text { National Stream Quality Accounting Network } \\ \text { USGS } & \text { U.S. Geological Survey } \\ \text { UR } & \text { Upriver (from noted location) }\end{array}$

\section{Frequency Symbols Used}

$\begin{array}{ll}\text { A } & \text { annually } \\ \text { BE } & \text { biennially (every 2 years) } \\ \text { BW } & \text { biweekly (every 2 weeks) } \\ \text { M } & \text { monthly } \\ \text { M Comp. } & \text { monthly composite } \\ \text { Q } & \text { quarterly } \\ \text { Q Comp. } & \text { quarterly composite } \\ \text { SA } & \text { semiannually (twice each year) } \\ \text { TE } & \text { triennially (every 3 years) }\end{array}$

\section{Analytical Symbols Used}

Generally, standard element, chemical, and isotope designations are used to indicate the analyses performed. Other analytical designations used include the following:

\begin{tabular}{|c|c|}
\hline Alpha & gross alpha activity of sample \\
\hline Anions & major anions - generally chloride, fluoride, nitrate, nitrite, sulfate \\
\hline Beta & gross beta activity of sample \\
\hline Gamma Scan & $\begin{array}{l}\text { analysis of photon energy spectrum for individual photon-emitting } \\
\text { radionuclides }\end{array}$ \\
\hline HTO & tritiated water $\left({ }^{3} \mathrm{H}^{1} \mathrm{H}^{16} \mathrm{O}\right)$ \\
\hline Hg-CVAA & mercury by cold vapor atomic absorbance spectrometry \\
\hline Hg-CVAF & total mercury in water by cold vapor atomic fluorescence \\
\hline ICP-MS & $\begin{array}{l}\text { major metals by inductively coupled plasma mass spectrometry - samples unfiltered } \\
\text { unless otherwise noted }\end{array}$ \\
\hline $\mathrm{Lo}^{3} \mathrm{H}$ & analytical procedure includes electrolytic enrichment \\
\hline $\mathrm{Pu}$ & isotopic plutonium $\left({ }^{238} \mathrm{Pu},{ }^{239 / 240} \mathrm{Pu}\right)$ \\
\hline
\end{tabular}


TOC total organic carbon

$\mathrm{U} \quad$ isotopic uranium $\left({ }^{234} \mathrm{U},{ }^{235} \mathrm{U},{ }^{238} \mathrm{U}\right)$

VOA volatile organic compounds 


\section{Contents}

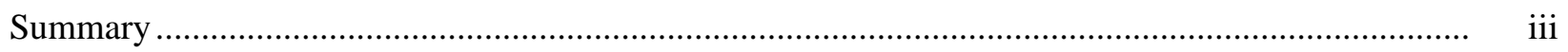

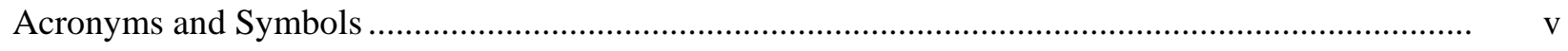

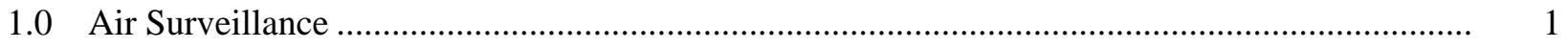

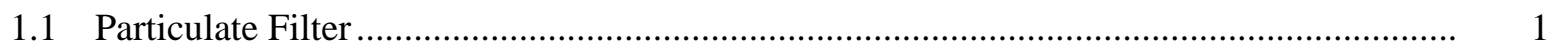

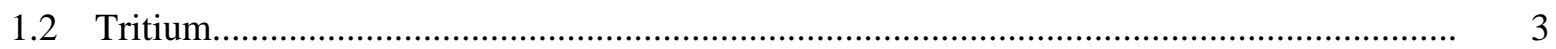

2.0 Surface Water Surveillance ............................................................................................

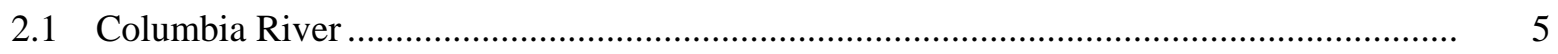

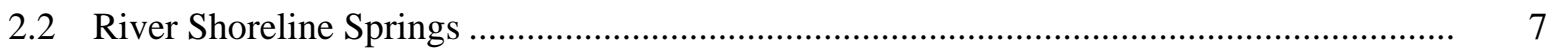

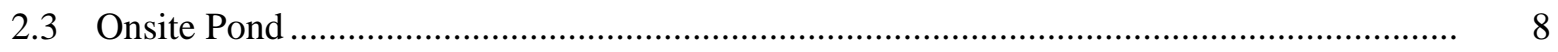

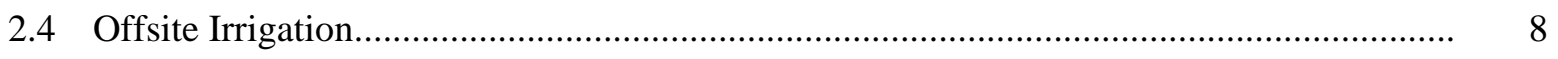

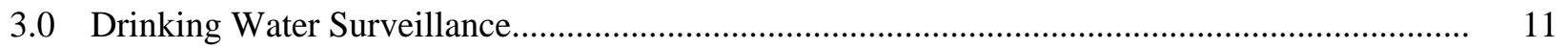

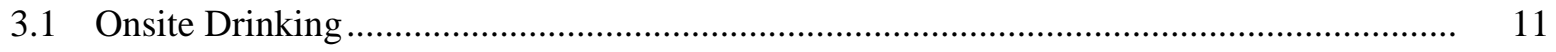

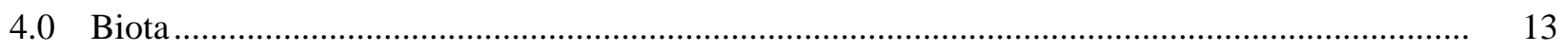

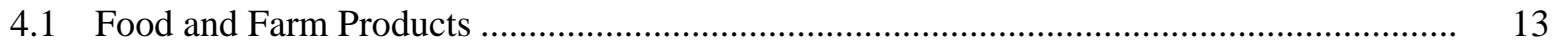

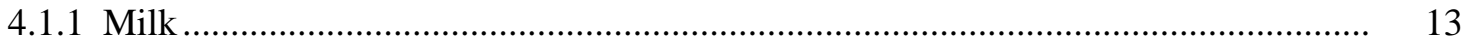

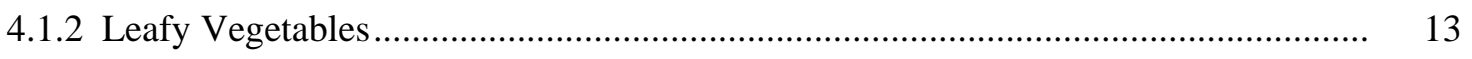

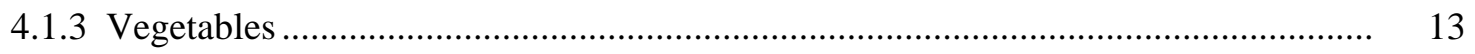

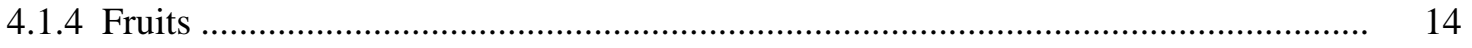

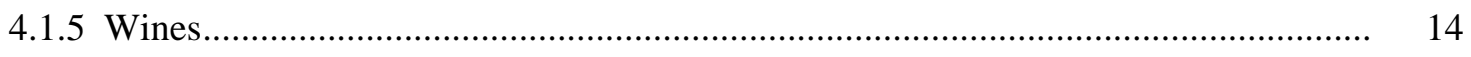

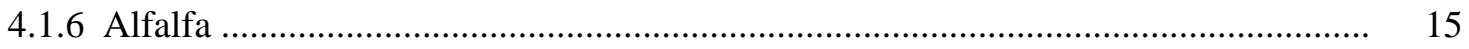

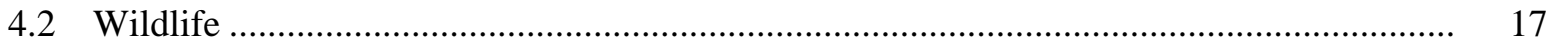

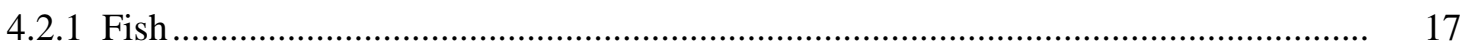

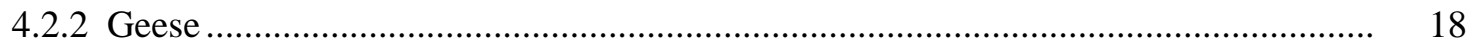

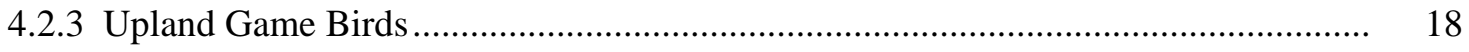

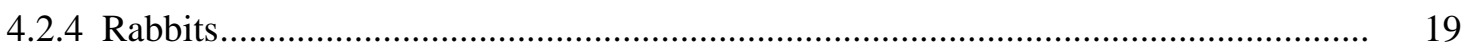

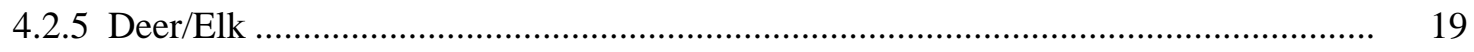

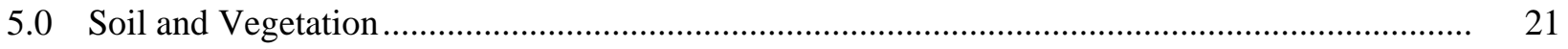

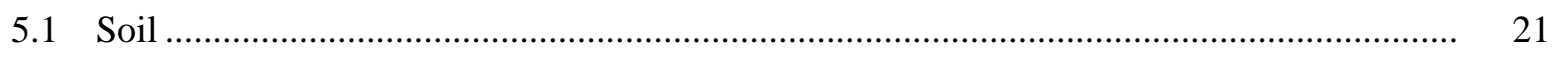

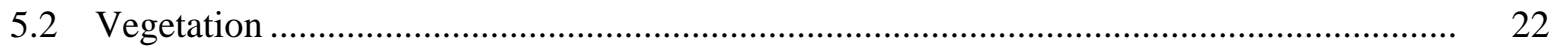

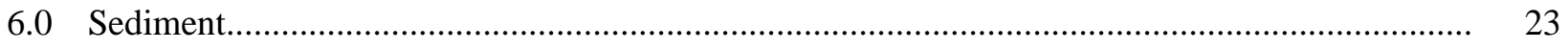

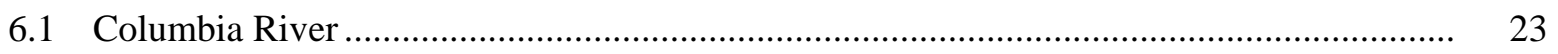

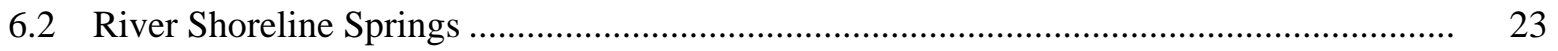

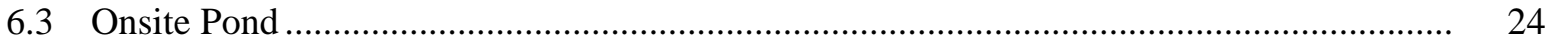

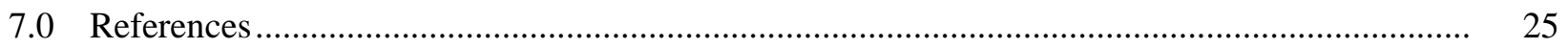




\section{Figures}

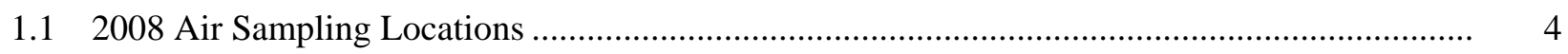

2.12008 Surface Water and Drinking Water Sampling Locations .................................................. 9

4.12008 Food and Farm Products Sampling Locations ….............................................................. 16

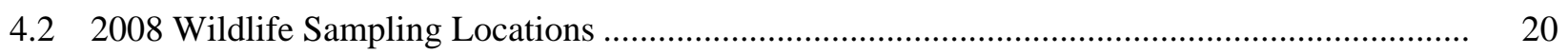

6.12008 Sediment Sampling Locations....................................................................................... 24 


\subsection{Air Surveillance}

\subsection{Particulate Filter}

\begin{tabular}{|c|c|c|c|c|c|c|}
\hline \multirow{2}{*}{ Location } & \multicolumn{3}{|c|}{ Individual Samples } & \multicolumn{3}{|c|}{ Composited Samples } \\
\hline & $\begin{array}{l}\text { Location } \\
\text { Number }^{(a)}\end{array}$ & $\begin{array}{l}\text { Fre- } \\
\text { quency }\end{array}$ & Analyses & Composite Group & $\begin{array}{l}\text { Fre- } \\
\text { quency }\end{array}$ & Analyses \\
\hline \multicolumn{7}{|l|}{ Onsite } \\
\hline 100 K Area & 1 & BW & Beta, Alpha & \multirow{3}{*}{100 Areas } & \multirow{3}{*}{ Q } & \multirow{3}{*}{${ }^{90} \mathrm{Sr}, \mathrm{Pu}, \mathrm{Gamma}$ Scan } \\
\hline 100 N-1325 Crib & 2 & BW & Beta, Alpha & & & \\
\hline 100 D Area & 3 & BW & Beta, Alpha & & & \\
\hline 100 F Met Tower & 4 & BW & Beta, Alpha & \multirow{2}{*}{ Hanford Townsite } & \multirow{2}{*}{$\mathrm{Q}$} & \multirow{2}{*}{${ }^{90} \mathrm{Sr}, \mathrm{Pu}, \mathrm{Gamma}$ Scan } \\
\hline Hanford Townsite & 5 & BW & Beta, Alpha & & & \\
\hline Gable Mountain & 6 & BW & Beta, Alpha & Gable Mountain & $\mathrm{Q}$ & Gamma Scan \\
\hline 200 ESE & 7 & BW & Beta, Alpha & \multirow{2}{*}{200 E Area } & \multirow{2}{*}{$\mathrm{Q}$} & \multirow{2}{*}{${ }^{90} \mathrm{Sr}, \mathrm{Pu}, \mathrm{U}, \mathrm{Gamma}$ Scan } \\
\hline S of $200 \mathrm{E}$ & 8 & BW & Beta, Alpha & & & \\
\hline B Pond & 9 & BW & Beta, Alpha & B Pond & Q & Pu, U, Gamma Scan \\
\hline Army Loop Camp & 10 & BW & Beta, Alpha & \multirow{3}{*}{200 W South East } & \multirow{3}{*}{$\mathrm{Q}$} & \multirow{3}{*}{${ }^{90} \mathrm{Sr}, \mathrm{Pu}, \mathrm{U}$, Gamma Scan } \\
\hline 200 Tel. Exchange & 11 & BW & Beta, Alpha & & & \\
\hline SW of B/C Cribs & 12 & BW & Beta, Alpha & & & \\
\hline 200 W SE & 13 & BW & Beta, Alpha & 200 West Area & $\mathrm{Q}$ & Pu, U, Gamma Scan \\
\hline 300 Water Intake & 14 & BW & Beta, Alpha & \multirow{3}{*}{300 Area } & \multirow{3}{*}{$\mathrm{Q}$} & \multirow{3}{*}{${ }^{90} \mathrm{Sr}, \mathrm{Pu}, \mathrm{U}$, Gamma Scan } \\
\hline 300 South Gate & 15 & BW & Beta, Alpha & & & \\
\hline 300 South West & 16 & BW & Beta, Alpha & & & \\
\hline 300 Trench & 17 & BW & Beta, Alpha & \multirow{2}{*}{$300 \mathrm{NE}$} & \multirow{2}{*}{ Q } & \multirow{2}{*}{${ }^{90} \mathrm{Sr}, \mathrm{Pu}, \mathrm{U}$, Gamma Scan } \\
\hline $300 \mathrm{NE}$ & 18 & BW & Beta, Alpha & & & \\
\hline $400 \mathrm{E}$ & 19 & BW & Beta, Alpha & \multirow{4}{*}{400 Area } & \multirow{4}{*}{$\mathrm{Q}$} & \multirow{4}{*}{${ }^{90} \mathrm{Sr}, \mathrm{Pu}, \mathrm{Gamma}$ Scan } \\
\hline $400 \mathrm{~W}$ & 20 & BW & Beta, Alpha & & & \\
\hline $400 \mathrm{~S}$ & 21 & BW & Beta, Alpha & & & \\
\hline $400 \mathrm{~N}$ & 22 & BW & Beta, Alpha & & & \\
\hline Wye Barricade ${ }^{(b)}$ & 23 & BW & Beta, Alpha & Wye Barricade & Q & ${ }^{90} \mathrm{Sr}, \mathrm{Pu}, \mathrm{U}$, Gamma Scan \\
\hline \multicolumn{7}{|l|}{$\underline{\text { Perimeter }}$} \\
\hline Ringold Met Tower & 24 & BW & Beta, Alpha & Ringold Met Tower & Q & ${ }^{90} \mathrm{Sr}, \mathrm{Pu}$, Gamma Scan \\
\hline W End of Fir Road ${ }^{(b)}$ & 25 & BW & Beta, Alpha & W End of Fir Road & Q & ${ }^{90} \mathrm{Sr}, \mathrm{Pu}, \mathrm{U}$, Gamma Scan \\
\hline
\end{tabular}


Particulate Filter (contd)

\begin{tabular}{|c|c|c|c|c|c|c|}
\hline \multirow[b]{2}{*}{ Location } & \multicolumn{3}{|c|}{ Individual Samples } & \multicolumn{3}{|c|}{ Composited Samples } \\
\hline & $\begin{array}{l}\text { Location } \\
\text { Number }^{(a)} \\
\end{array}$ & $\begin{array}{l}\text { Fre- } \\
\text { quency }\end{array}$ & Analyses & Composite Group & $\begin{array}{l}\text { Fre- } \\
\text { quency }\end{array}$ & Analyses \\
\hline \multicolumn{7}{|l|}{$\underline{\text { Perimeter }}$} \\
\hline Dogwood Met Tower & 26 & BW & Beta, Alpha & Dogwood Met Tower & Q & ${ }^{90} \mathrm{Sr}, \mathrm{U}, \mathrm{Gamma}$ Scan \\
\hline Byers Landing & 27 & BW & Beta, Alpha & Byers Landing & Q & ${ }^{90} \mathrm{Sr}, \mathrm{Pu}, \mathrm{U}, \mathrm{Gamma}$ Scan \\
\hline Battelle Complex ${ }^{(b)}$ & 28 & BW & Beta, Alpha & Battelle Complex & Q & U, Gamma Scan \\
\hline $\begin{array}{l}\text { Horn Rapids Substa } \\
\text { Prosser Barricade }{ }^{(b)}\end{array}$ & $\begin{array}{l}29 \\
30\end{array}$ & $\begin{array}{l}\text { BW } \\
\text { BW }\end{array}$ & $\left.\begin{array}{l}\text { Beta, Alpha } \\
\text { Beta, Alpha }\end{array}\right\}$ & Prosser Barricade & Q & ${ }^{90} \mathrm{Sr}, \mathrm{Pu}, \mathrm{Gamma}$ Scan \\
\hline $\begin{array}{l}\text { Yakima Barricade }{ }^{(b)} \\
\text { Rattlesnake Springs }\end{array}$ & $\begin{array}{l}31 \\
32\end{array}$ & $\begin{array}{l}\text { BW } \\
\text { BW }\end{array}$ & $\left.\begin{array}{l}\text { Beta, Alpha } \\
\text { Beta, Alpha }\end{array}\right\}$ & Yakima Barricade & $\mathrm{Q}$ & ${ }^{90} \mathrm{Sr}, \mathrm{Pu}, \mathrm{Gamma}$ Scan \\
\hline $\begin{array}{l}\text { Wahluke Slope } \\
\text { S End Vernita Bridge }\end{array}$ & $\begin{array}{l}33 \\
34\end{array}$ & $\begin{array}{l}\text { BW } \\
\text { BW }\end{array}$ & $\left.\begin{array}{l}\text { Beta, Alpha } \\
\text { Beta, Alpha }\end{array}\right\}$ & Wahluke Slope & Q & ${ }^{90} \mathrm{Sr}, \mathrm{Pu}, \mathrm{Gamma}$ Scan \\
\hline \multicolumn{7}{|l|}{ Community } \\
\hline Basin City School & 35 & BW & Beta, Alpha & Basin City School & Q & ${ }^{90} \mathrm{Sr}$, Pu, U, Gamma Scan \\
\hline Leslie Groves-Rchlnd & 36 & BW & Beta, Alpha & Leslie Groves-Rchlnd & Q & ${ }^{90} \mathrm{Sr}, \mathrm{Pu}, \mathrm{U}$, Gamma Scan \\
\hline $\begin{array}{l}\text { Pasco } \\
\text { Kennewick-Ely Street }\end{array}$ & $\begin{array}{l}37 \\
38\end{array}$ & $\begin{array}{l}\text { BW } \\
\text { BW }\end{array}$ & $\begin{array}{l}\text { Beta } \\
\text { Beta, Alpha }\end{array}$ & Tri Cities & Q & ${ }^{90} \mathrm{Sr}, \mathrm{Pu}, \mathrm{U}$, Gamma Scan \\
\hline Benton City & 39 & BW & Beta & Benton City & $\mathrm{Q}$ & Gamma Scan \\
\hline Mattawa & 40 & BW & Beta & Mattawa & Q & Gamma Scan \\
\hline Othello & 41 & BW & Beta & Othello & Q & Gamma Scan \\
\hline \multicolumn{7}{|l|}{ Distant } \\
\hline Yakima & 42 & BW & Beta, Alpha & Yakima & Q & ${ }^{90} \mathrm{Sr}, \mathrm{Pu}, \mathrm{U}$, Gamma Scan \\
\hline
\end{tabular}

(a) Refer to Figure 1.1, “2008 Air Sampling Locations.”

(b) Washington State Department of Health (DOH) air sampler also at this location. 


\subsection{Tritium}

\begin{tabular}{|c|c|c|c|}
\hline Location & $\begin{array}{l}\text { Location } \\
\text { Number }^{(a)}\end{array}$ & Frequency & Analysis $^{(b)}$ \\
\hline \multicolumn{4}{|l|}{$\underline{\text { Onsite }}$} \\
\hline 100 K Area & 1 & M & ${ }^{3} \mathrm{H}$ \\
\hline 100 N-1325 Crib & 2 & M & ${ }^{3} \mathrm{H}$ \\
\hline $200 \mathrm{ESE}$ & 7 & M & ${ }^{3} \mathrm{H}$ \\
\hline 200 Tel. Exchange & 11 & M & ${ }^{3} \mathrm{H}$ \\
\hline 300 Water Intake ${ }^{(c)}$ & 14 & M & ${ }^{3} \mathrm{H}$ \\
\hline 300 South Gate ${ }^{(\mathrm{d})}$ & 15 & M & ${ }^{3} \mathrm{H}$ \\
\hline 300 South West & 16 & M & ${ }^{3} \mathrm{H}$ \\
\hline 300 Trench & 17 & M & ${ }^{3} \mathrm{H}$ \\
\hline $300 \mathrm{NE}$ & 18 & M & ${ }^{3} \mathrm{H}$ \\
\hline $400 \mathrm{E}$ & 19 & M & ${ }^{3} \mathrm{H}$ \\
\hline
\end{tabular}

Perimeter

$\begin{array}{llll}\text { Ringold Met Tower } & 24 & \text { M } & { }^{3} \mathrm{H} \\ \text { W End of Fir Road } & 25 & \text { M } & { }^{3} \mathrm{H} \\ \text { Dogwood Met Tower } & 26 & \text { M } & { }^{3} \mathrm{H} \\ \text { Byers Landing } & 27 & \text { M } & { }^{3} \mathrm{H} \\ \text { Battelle Complex } & \left.{ }^{3}\right) & \text { M } & { }^{3} \mathrm{H} \\ \text { Prosser Barricade } & 28 & \text { M } & { }^{3} \mathrm{H} \\ \text { Wahluke Slope } & 30 & \text { M } & { }^{3} \mathrm{H}\end{array}$

Community

Basin City School

$35 \quad \mathrm{M} \quad{ }^{3} \mathrm{H}$

Leslie Groves-Rchlnd $\quad 36 \quad M \quad{ }^{3} \mathrm{H}$

$\underline{\text { Distant }}$

Yakima

42

M

${ }^{3} \mathrm{H}$

(a) Refer to Figure 1.1, “2008 Air Sampling Locations.”

(b) As tritiated water (HTO).

(c) $\mathrm{DOH}$ air sampler also at this location.

(d) Two tritium samples are collected from this location. 


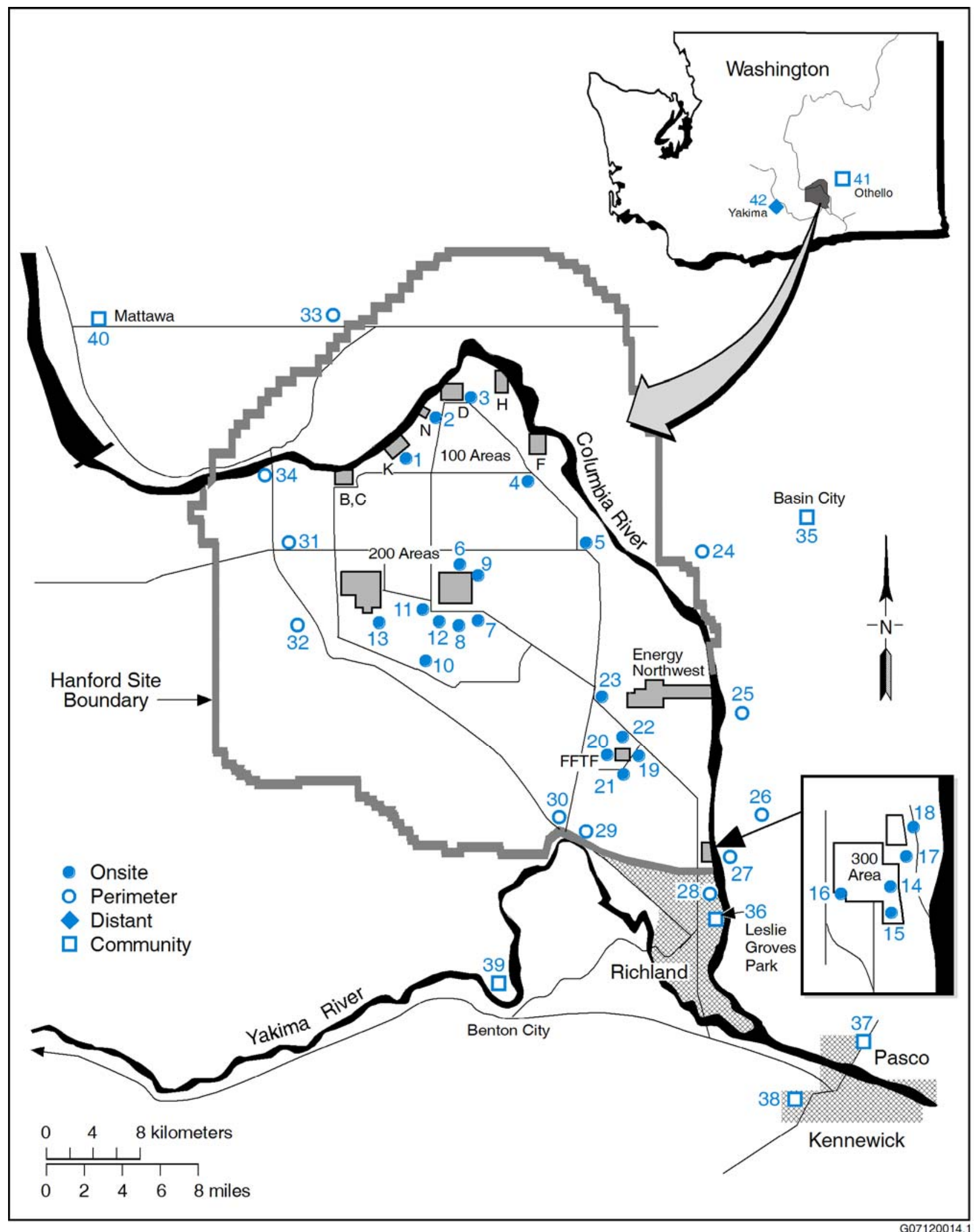

Figure 1.1. 2008 Air Sampling Locations 


\subsection{Surface Water Surveillance}

\subsection{Columbia River}

\begin{tabular}{|c|c|c|c|}
\hline Location $^{(a)}$ & $\underline{\text { Sample Type }}$ & Frequency & Analyses/Agency \\
\hline Priest Rapids-River & Cumulative & M Comp. ${ }^{(b)}$ & Alpha, Beta, Lo ${ }^{3} \mathrm{H},{ }^{90} \mathrm{Sr},{ }^{99} \mathrm{Tc}, \mathrm{U} / \mathrm{DOH}^{(\mathrm{c})}$ \\
\hline & Particulate (filter) & $\begin{array}{l}\text { M Comp. } \\
\text { Q Comp. }^{(\mathrm{d})}\end{array}$ & $\begin{array}{l}\text { Gamma Scan } \\
\mathrm{Pu}\end{array}$ \\
\hline & Soluble (resin) & $\begin{array}{l}\text { M Comp. } \\
\text { Q Comp. }^{(\mathrm{d})}\end{array}$ & $\begin{array}{l}\text { Gamma Scan } \\
\mathrm{Pu}\end{array}$ \\
\hline Rich.Pmphs HRM 46.4 & Cumulative & M Comp. ${ }^{(b)}$ & Alpha, Beta, Lo ${ }^{3} \mathrm{H},{ }^{90} \mathrm{Sr},{ }^{99} \mathrm{Tc}, \mathrm{U}$ \\
\hline & Particulate (filter) & $\begin{array}{l}\text { M Comp. } \\
\text { Q Comp. }^{(\mathrm{d})}\end{array}$ & $\begin{array}{l}\text { Gamma Scan } \\
\mathrm{Pu}\end{array}$ \\
\hline & Soluble (resin) & $\begin{array}{l}\text { M Comp. } \\
\text { Q Comp. }^{(\mathrm{d})}\end{array}$ & $\begin{array}{l}\text { Gamma Scan } \\
\mathrm{Pu}\end{array}$ \\
\hline & Grab & 3/Yr & USGS-NASQAN $^{(\mathrm{e})}$ \\
\hline Rich.Pmphs-1 HRM46.4 ${ }^{(\mathrm{f})}$ & Transect & $\begin{array}{l}\text { Q } \\
\text { A }\end{array}$ & $\begin{array}{l}\text { Lo }{ }^{3} \mathrm{H},{ }^{90} \mathrm{Sr}, \mathrm{U} \text {, Anions } \\
\text { ICP-MS, Ho-CVAF ICP-MS Filtered VOA }\end{array}$ \\
\hline Rich.Pmphs-2 HRM46.4 & Transect & $\mathrm{Q}$ & $\begin{array}{l}\text { Lo }{ }^{3} \mathrm{H},{ }^{90} \mathrm{Sr}, \mathrm{U}, \text { Anions } \\
\text { ICP-MS, Hg-CVAF, ICP-MS Filtered, VOA }\end{array}$ \\
\hline Rich.Pmphs-3 HRM46.4 & Transect & $\begin{array}{l}\mathrm{Q} \\
\mathrm{A}\end{array}$ & $\begin{array}{l}\text { Lo }{ }^{3} \mathrm{H},{ }^{90} \mathrm{Sr}, \mathrm{U}, \text { Anions } \\
\text { ICP-MS, Hg-CVAF, ICP-MS Filtered, VOA }\end{array}$ \\
\hline Rich.Pmphs-5 HRM46.4 & Transect & $\begin{array}{l}\mathrm{Q} \\
\mathrm{A}\end{array}$ & $\begin{array}{l}\text { Lo }{ }^{3} \mathrm{H},{ }^{90} \mathrm{Sr}, \mathrm{U}, \text { Anions } \\
\text { ICP-MS, Hg-CVAF, ICP-MS Filtered, VOA }\end{array}$ \\
\hline Rich.Pmphs-7 HRM46.4 & Transect & Q & $\begin{array}{l}\text { Lo }{ }^{3} \mathrm{H},{ }^{90} \mathrm{Sr}, \mathrm{U}, \text { Anions } \\
\text { ICP-MS, Hg-CVAF, ICP-MS Filtered, VOA }\end{array}$ \\
\hline Rich.Pmphs-10 HRM46.4 & Transect & $\mathrm{Q}$ & $\begin{array}{l}\text { Lo }{ }^{3} \mathrm{H},{ }^{90} \mathrm{Sr}, \mathrm{U}, \text { Anions } \\
\text { ICP-MS, Hg-CVAF, ICP-MS Filtered, VOA }\end{array}$ \\
\hline Rich.Pmphs HRM 43.5 & Transect & $\mathrm{Q}$ & $\begin{array}{l}\text { Lo }{ }^{3} \mathrm{H},{ }^{90} \mathrm{Sr}, \mathrm{U}, \text { Anions } \\
\text { ICP-MS, Hg-CVAF, ICP-MS Filtered }\end{array}$ \\
\hline Rich.Pmphs HRM 43.9 & Transect & $\begin{array}{l}\mathrm{Q} \\
\mathrm{A}\end{array}$ & $\begin{array}{l}\text { Lo }{ }^{3} \mathrm{H},{ }^{90} \mathrm{Sr}, \mathrm{U} \text {, Anions } \\
\text { ICP-MS, Hg-CVAF, ICP-MS Filtered }\end{array}$ \\
\hline Rich.Pmphs HRM 45.0 & Transect & $\mathrm{Q}$ & $\begin{array}{l}\text { Lo }{ }^{3} \mathrm{H},{ }^{90} \mathrm{Sr}, \mathrm{U} \text {, Anions } \\
\text { ICP-MS, Hg-CVAF, ICP-MS Filtered }\end{array}$ \\
\hline Rich.Pmphs HRM 45.8 & Transect & $\mathrm{Q}$ & $\begin{array}{l}\mathrm{Lo}{ }^{3} \mathrm{H},{ }^{90} \mathrm{Sr}, \mathrm{U}, \text { Anions } \\
\text { ICP-MS, Hg-CVAF, ICP-MS Filtered }\end{array}$ \\
\hline Vernita & Grab & 3/Yr & USGS-NASQAN ${ }^{(\mathrm{e})}$ \\
\hline Vernita-1 HRM 0.3 & Transect & $\begin{array}{l}\mathrm{Q} \\
\mathrm{A}\end{array}$ & $\begin{array}{l}\text { Lo }{ }^{3} \mathrm{H},{ }^{90} \mathrm{Sr}, \mathrm{U}, \text { Anions } \\
\text { ICP-MS, Hg-CVAF, ICP-MS Filtered, VOA }\end{array}$ \\
\hline Vernita-2 HRM 0.3 & Transect & $\begin{array}{l}\mathrm{Q} \\
\mathrm{A}\end{array}$ & $\begin{array}{l}\text { Lo }{ }^{3} \mathrm{H},{ }^{90} \mathrm{Sr}, \mathrm{U} \text {, Anions } \\
\text { ICP-MS, Hg-CVAF. ICP-MS Filtered, VOA }\end{array}$ \\
\hline Vernita-3 HRM 0.3 & Transect & $\begin{array}{l}\mathrm{Q} \\
\mathrm{A}\end{array}$ & $\begin{array}{l}\text { Lo 3H, 90Sr, U, Anions } \\
\text { ICP-MS, Hg-CVAF, ICP-MS Filtered, VOA }\end{array}$ \\
\hline Vernita-4 HRM 0.3 & Transect & $\begin{array}{l}\mathrm{Q} \\
\mathrm{A}\end{array}$ & $\begin{array}{l}\text { Lo } 3 \mathrm{H}, 90 \mathrm{Sr}, \mathrm{U} \text {, Anions } \\
\text { ICP-MS, Hg-CVAF, ICP-MS Filtered, VOA }\end{array}$ \\
\hline
\end{tabular}




\section{Columbia River (contd)}

\begin{tabular}{l} 
Location $^{(\mathrm{a})}$ \\
\hline 100 N -1 HRM 9.5 \\
(g) \\
100 N -2 HRM 9.5 \\
100 N -3 HRM 9.5 \\
100 N -5 HRM 9.5 \\
100 N -7 HRM 9.5 \\
100 N -10 HRM 9.5 \\
100 N Shore HRM 8.4 \\
100 N Shore HRM 8.9 \\
100 N Shore HRM 9.2 \\
100 N Shore HRM 9.8 \\
Hanfrd TS-1 HRM 28.7 \\
Hanfrd TS-2 HRM 28.7 \\
Hanfrd TS-3 HRM 28.7 \\
Hanfrd TS-5 HRM 28.7 \\
Hanfrd TS-7 HRM 28.7 \\
Hanfrd TS-10 HRM 28.7 \\
Hanfrd Twnsite HRM26 \\
Hanfrd Twnsite HRM27 \\
Hanfrd Twnsite HRM28 \\
Hanfrd Twnsite HRM30 \\
300 Area -1 HRM 43.1 \\
300 Area -2 HRM 43.1 \\
300 Area -3 HRM 43.1 \\
300 Area -5 HRM 43.1 \\
300 Area -7 HRM 43.1 \\
300 Area -10 HRM 43.1 \\
300 Area Shr HRM41.5 \\
300 Area Spring $42-2$ \\
\end{tabular}

300 Area Outfl13

\begin{tabular}{cr} 
Sample Type & Freq \\
\hline Transect & A \\
Transect & A \\
Transect & A \\
Transect & A \\
Transect & A \\
Transect & A \\
Transect & A \\
Transect & A \\
Transect & A \\
Transect & A \\
Transect & \\
Transect & A \\
Transect & A \\
Transect & A \\
Transect & A \\
Transect & A \\
Transect & A \\
Transect & A \\
Transect & A \\
Transect & A \\
Transect & A \\
Transect & A \\
Transect & A \\
Transect & A \\
Transect & A \\
Transect & A \\
Transect & A \\
Trabsect & A \\
Trans &
\end{tabular}

\section{requency}

A

A

A

A

A

A

A

A

A

A

A

A

A

A

A

A

A

A

A

A

A

A

A

A

A

A

A

A

A

A

Q
Analyses/Agency

Lo ${ }^{3} \mathrm{H},{ }^{90} \mathrm{Sr}$, U, ICP-MS, ICP-MS Filtered, Anions Lo ${ }^{3} \mathrm{H},{ }^{90} \mathrm{Sr}$, U, ICP-MS, ICP-MS Filtered, Anions Lo ${ }^{3} \mathrm{H},{ }^{90} \mathrm{Sr}$, U, ICP-MS, ICP-MS Filtered, Anions Lo ${ }^{3} \mathrm{H},{ }^{90} \mathrm{Sr}$, U, ICP-MS, ICP-MS Filtered, Anions Lo ${ }^{3} \mathrm{H},{ }^{90} \mathrm{Sr}$, U, ICP-MS, ICP-MS Filtered, Anions Lo ${ }^{3} \mathrm{H},{ }^{90} \mathrm{Sr}$, U, ICP-MS, ICP-MS Filtered, Anions Lo ${ }^{3} \mathrm{H},{ }^{90} \mathrm{Sr}$, U, ICP-MS, ICP-MS Filtered, Anions/DOH ${ }^{(\mathrm{h})}$ Lo ${ }^{3} \mathrm{H},{ }^{90} \mathrm{Sr}$, U, ICP-MS, ICP-MS Filtered, Anions/DOH ${ }^{(\mathrm{h})}$ Lo ${ }^{3} \mathrm{H},{ }^{90} \mathrm{Sr}$, U, ICP-MS, ICP-MS Filtered, Anions/DOH ${ }^{(\mathrm{h})}$ Lo ${ }^{3} \mathrm{H},{ }^{90} \mathrm{Sr}$, U, ICP-MS, ICP-MS Filtered, Anions/DOH ${ }^{(\mathrm{h})}$ Lo ${ }^{3} \mathrm{H},{ }^{90} \mathrm{Sr},{ }^{99} \mathrm{Tc}, \mathrm{U}$, ICP-MS, ICP-MS Filtered, Anions Lo ${ }^{3} \mathrm{H},{ }^{90} \mathrm{Sr}$, U, ICP-MS, ICP-MS Filtered, Anions Lo ${ }^{3} \mathrm{H},{ }^{90} \mathrm{Sr}$, U, ICP-MS, ICP-MS Filtered, Anions Lo ${ }^{3} \mathrm{H},{ }^{90} \mathrm{Sr}$, U, ICP-MS, ICP-MS Filtered, Anions Lo ${ }^{3} \mathrm{H},{ }^{90} \mathrm{Sr}$, U, ICP-MS, ICP-MS Filtered, Anions Lo ${ }^{3} \mathrm{H},{ }^{90} \mathrm{Sr}$, U, ICP-MS, ICP-MS Filtered, Anions Lo ${ }^{3} \mathrm{H},{ }^{90} \mathrm{Sr},{ }^{99} \mathrm{Tc}, \mathrm{U}$, ICP-MS, ICP-MS Filtered, Anions/DOH ${ }^{(\mathrm{h})}$ Lo ${ }^{3} \mathrm{H},{ }^{90} \mathrm{Sr},{ }^{99} \mathrm{Tc}, \mathrm{U}$, ICP-MS, ICP-MS Filtered, Anions/DOH ${ }^{(\mathrm{h})}$ Lo ${ }^{3} \mathrm{H},{ }^{90} \mathrm{Sr},{ }^{99} \mathrm{Tc}, \mathrm{U}, \mathrm{ICP}-\mathrm{MS}$, ICP-MS Filtered, Anions/DOH ${ }^{(\mathrm{h})}$ Lo ${ }^{3} \mathrm{H},{ }^{90} \mathrm{Sr},{ }^{99} \mathrm{Tc}, \mathrm{U}$, ICP-MS, ICP-MS Filtered, Anions/DOH ${ }^{(\mathrm{h})}$ Lo ${ }^{3} \mathrm{H},{ }^{90} \mathrm{Sr}, \mathrm{U}$, ICP-MS, ICP-MS Filtered, Anions Lo ${ }^{3} \mathrm{H},{ }^{90} \mathrm{Sr}$, U, ICP-MS, ICP-MS Filtered, Anions Lo ${ }^{3} \mathrm{H},{ }^{90} \mathrm{Sr}$, U, ICP-MS, ICP-MS Filtered, Anions Lo ${ }^{3} \mathrm{H},{ }^{90} \mathrm{Sr}$, U, ICP-MS, ICP-MS Filtered, Anions Lo ${ }^{3} \mathrm{H},{ }^{90} \mathrm{Sr}$, U, ICP-MS, ICP-MS Filtered, Anions Lo ${ }^{3} \mathrm{H},{ }^{90} \mathrm{Sr}$, U, ICP-MS, ICP-MS Filtered, Anions Lo ${ }^{3} \mathrm{H},{ }^{90} \mathrm{Sr}$, U, ICP-MS, ICP-MS Filtered, Anions/DOH ${ }^{(\mathrm{h})}$ Lo ${ }^{3} \mathrm{H},{ }^{90} \mathrm{Sr}$, U, ICP-MS, ICP-MS Filtered, Anions/DOH ${ }^{(\mathrm{h})}$ Lo ${ }^{3} \mathrm{H},{ }^{90} \mathrm{Sr}$, U, ICP-MS, ICP-MS Filtered, Anions/DOH ${ }^{(\mathrm{h})}$ Lo ${ }^{3} \mathrm{H},{ }^{90} \mathrm{Sr}$, U, ICP-MS, ICP-MS Filtered, Anions/DOH ${ }^{(\mathrm{h})}$

Lo ${ }^{3} \mathrm{H},{ }^{90} \mathrm{Sr}, \mathrm{U}$, Anions ICP-MS, ICP-MS Filtered

ICP-MS = Inductively coupled plasma mass spectrometry. VOA = Volatile organic analysis.

(a) Refer to Figure 2.1, "2008 Surface Water and Drinking Water Sampling Locations.” Hanford river markers (HRM) are a series of signposts along the Hanford Site shoreline of the Columbia River that are roughly 1.6 kilometer (1 mile) apart. The Vernita Bridge is HRM \#0 and Ferry Street in Richland is HRM \#46. Samples collected between HRMs are assigned a decimal.

(b) Sample is collected weekly and composited monthly for analysis.

(c) Additional sample provided to the DOH (January and June only).

(d) Sample is collected biweekly and composited for analysis.

(e) Analyses are performed by the U.S. Geological Survey (USGS) in conjunction with the National Stream Quality Accounting Network (NASQAN) Program, and include conductance, $\mathrm{pH}$, temperature, turbidity, dissolved oxygen, hardness, Ca, Mg, alkalinity, carbonates, sulfate, $\mathrm{Cl}, \mathrm{F}$, solids, $\mathrm{NH}_{4}-\mathrm{N}, \mathrm{NO}_{3}+\mathrm{NO}_{2}$, N-Kjeldahl, $\mathrm{P}, \mathrm{Cr}$, Fe, and dissolved organic carbon.

(f) Quality assurance sample submitted for analyses twice per year.

(g) Quality assurance sample submitted for analyses once per year.

(h) Additional sample provided to the $\mathrm{DOH}$. 


\subsection{River Shoreline Springs}

\begin{tabular}{|c|c|c|}
\hline Location $^{(a)}$ & $\underline{\mathrm{HRM}}^{(\mathrm{b})}$ & $\begin{array}{c}\text { Sample } \\
\text { Type }\end{array}$ \\
\hline 100-B Spring 38-3 & 3.8 & Grab \\
\hline 100-B Spring 39-2 & 3.9 & Grab \\
\hline 100-K Spring 63-1 & 6.3 & Grab \\
\hline 100-K Spring 77-1 & 7.6 & Grab \\
\hline 100-N Spring 8-13 & 9.3 & Grab \\
\hline 100-D Spring 102-1 & 10.2 & Grab \\
\hline 100-D Spring 110-1 & 11.0 & Grab \\
\hline 100-H Spring 145-1 & 14.4 & Grab \\
\hline 100-H Spring 153-1 & 15.3 & Grab \\
\hline 100-F Spring 207-1 & 21.3 & Grab \\
\hline Hanford Spr UR 28-2 ${ }^{(\mathrm{d})}$ & 27.8 & Grab \\
\hline Hanford Spring 28-2 & 28.1 & Grab \\
\hline Hanford Spr DR 28-2 ${ }^{(\mathrm{e})}$ & 28.3 & Grab \\
\hline 300 Area Spring 41-9 & 41.9 & Grab \\
\hline 300 Area Spring 42-2 & 42.1 & Grab \\
\hline 300 Area Spr DR 42-2 ${ }^{(\mathrm{e})}$ & 42.4 & Grab \\
\hline 300 Area Spring 42-7 & 42.7 & Grab \\
\hline Richland Spr(SRL 437-1) & 43.7 & Grab \\
\hline
\end{tabular}

Analyses/Agency

Alpha, Beta, ${ }^{3} \mathrm{H},{ }^{90} \mathrm{Sr},{ }^{99} \mathrm{Tc}$, Gamma Scan, ICP-MS, Hg-CVAF, ICP-MS Filtered, Anions, VOA/DOH ${ }^{(\mathrm{c})}$

Alpha, Beta, ${ }^{3} \mathrm{H},{ }^{90} \mathrm{Sr},{ }^{99} \mathrm{Tc}$, Gamma Scan, ICP-MS, Hg-CVAF, ICP-MS Filtered, Anions, VOA/DOH ${ }^{(\mathrm{c})}$

Alpha, Beta, ${ }^{3} \mathrm{H},{ }^{90} \mathrm{Sr}$, Gamma Scan, ICP-MS, Hg-CVAF, ICP-MS Filtered, Anions, VOA

Alpha, Beta, ${ }^{3} \mathrm{H},{ }^{90} \mathrm{Sr}$, Gamma Scan, ICP-MS, Hg-CVAF, ICP-MS Filtered, Anions, VOA

Alpha, Beta, ${ }^{3} \mathrm{H},{ }^{90} \mathrm{Sr}$, Gamma Scan, ICP-MS, Hg-CVAF, ICP-MS Filtered, Anions/DOH ${ }^{(\mathrm{c})}$

Alpha, Beta, ${ }^{3} \mathrm{H},{ }^{90} \mathrm{Sr}$, Gamma Scan, ICP-MS, Hg-CVAF, ICP-MS Filtered, Anions/DOH ${ }^{(\mathrm{c})}$

Alpha, Beta, ${ }^{3} \mathrm{H},{ }^{90} \mathrm{Sr}$, Gamma Scan, ICP-MS, Hg-CVAF, ICP-MS Filtered, Anions/DOH ${ }^{(\mathrm{c})}$

Alpha, Beta, ${ }^{3} \mathrm{H},{ }^{90} \mathrm{Sr},{ }^{99} \mathrm{Tc}, \mathrm{U}, \mathrm{Gamma}$ Scan, ICP-MS, Hg-CVAF, ICP-MS Filtered, Anions

Alpha, Beta, ${ }^{3} \mathrm{H},{ }^{90} \mathrm{Sr},{ }^{99} \mathrm{Tc}, \mathrm{U}$, Gamma Scan, ICP-MS, Hg-CVAF, ICP-MS Filtered, Anions

Alpha, Beta, ${ }^{3} \mathrm{H},{ }^{90} \mathrm{Sr}$, U, Gamma Scan, ICP-MS, Hg-CVAF, ICP-MS Filtered, Anions, VOA

Alpha, Beta, ${ }^{3} \mathrm{H},{ }^{99} \mathrm{Tc}, \mathrm{U},{ }^{129} \mathrm{I}$, Gamma Scan, ICP-MS, Hg-CVAF, ICP-MS Filtered, Anions/ $\mathrm{DOH}^{(\mathrm{c})}$

Alpha, Beta, ${ }^{3} \mathrm{H},{ }^{99}$ Tc, U, ${ }^{129}$ I, Gamma Scan, ICP-MS, Hg-CVAF, ICP-MS Filtered, Anions/DOH ${ }^{(\mathrm{c})}$

Alpha, Beta, ${ }^{3} \mathrm{H},{ }^{99}$ Tc, U, ${ }^{129}$ I, Gamma Scan, ICP-MS, Hg-CVAF, ICP-MS Filtered, Anions/DOH ${ }^{(\mathrm{c})}$

Alpha, Beta, ${ }^{3} \mathrm{H},{ }^{90} \mathrm{Sr}, \mathrm{U}$, Gamma Scan, ICP-MS, Hg-CVAF, ICP-MS Filtered, Anions

Alpha, Beta, ${ }^{3} \mathrm{H},{ }^{90} \mathrm{Sr}, \mathrm{U},{ }^{129} \mathrm{I}$, Gamma Scan, ICP-MS, Hg-CVAF, ICP-MS Filtered, Anions, VOA/ $\mathrm{DOH}^{(\mathrm{c})}$

Alpha, Beta, ${ }^{3} \mathrm{H},{ }^{90} \mathrm{Sr}, \mathrm{U},{ }^{129} \mathrm{I}$, Gamma Scan, ICP-MS, Hg-CVAF, ICP-MS Filtered, Anions, VOA/ $\mathrm{DOH}^{(\mathrm{c})}$

Alpha, Beta, ${ }^{3} \mathrm{H},{ }^{90} \mathrm{Sr}, \mathrm{U}$, Gamma Scan, Anions

Alpha, Beta, ${ }^{3} \mathrm{H},{ }^{90} \mathrm{Sr}, \mathrm{U}$, Gamma Scan, ICP-MS, Hg-CVAF, ICP-MS Filtered, Anions

(a) Refer to Figure 2.1, "2008 Surface Water and Drinking Water Sampling Locations.”

(b) Hanford river markers (HRM) are a series of signposts along the Hanford Site shoreline of the Columbia River that are roughly 1.6 kilometers (1 mile) apart. The Vernita Bridge is HRM \#0 and Ferry Street in Richland is HRM \#46. Samples collected between HRMs are assigned a decimal.

(c) Additional sample provided to the $\mathrm{DOH}$.

(d) UR = Upriver from noted location.

(e) $\mathrm{DR}=$ Downriver from noted location. 


\subsection{Onsite Pond}

\begin{tabular}{|c|c|c|c|}
\hline Location $^{(\mathrm{a})}$ & Sample Type & Frequency & Analyses \\
\hline West Lake & Grab & Q & ${ }^{3} \mathrm{H}$ \\
\hline FFTF Pond ${ }^{(b)}$ & Grab & Q & Alpha, Beta, ${ }^{3} \mathrm{H}$, Gamma Scan \\
\hline
\end{tabular}

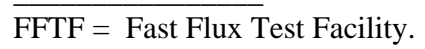

(a) Refer to Figure 2.1, "2008 Surface Water and Drinking Water Sampling Locations.”

(b) Quality assurance sample submitted for analyses once per year.

\subsection{Offsite Irrigation}

\begin{tabular}{|c|c|c|c|}
\hline Location $^{(\mathrm{a})}$ & $\underline{\text { Sample Type }}$ & Frequency & Analyses/Agency \\
\hline Riverview Canal & Grab & 3 (May-Sept) & Alpha, Beta, Lo ${ }^{3} \mathrm{H},{ }^{90} \mathrm{Sr}, \mathrm{U}, \mathrm{Gamma}$ Scan/DOH ${ }^{(\mathrm{b})}$ \\
\hline Horn Rapids Area & Grab & 3 (May-Sept) & Alpha, Beta, Lo ${ }^{3} \mathrm{H},{ }^{90} \mathrm{Sr}, \mathrm{U}, \mathrm{Gamma}$ Scan $/ \mathrm{DOH}^{(\mathrm{b})}$ \\
\hline
\end{tabular}

(a) Refer to Figure 2.1, “2008 Surface Water and Drinking Water Sampling Locations.”

(b) Additional sample provided to the $\mathrm{DOH}$. 


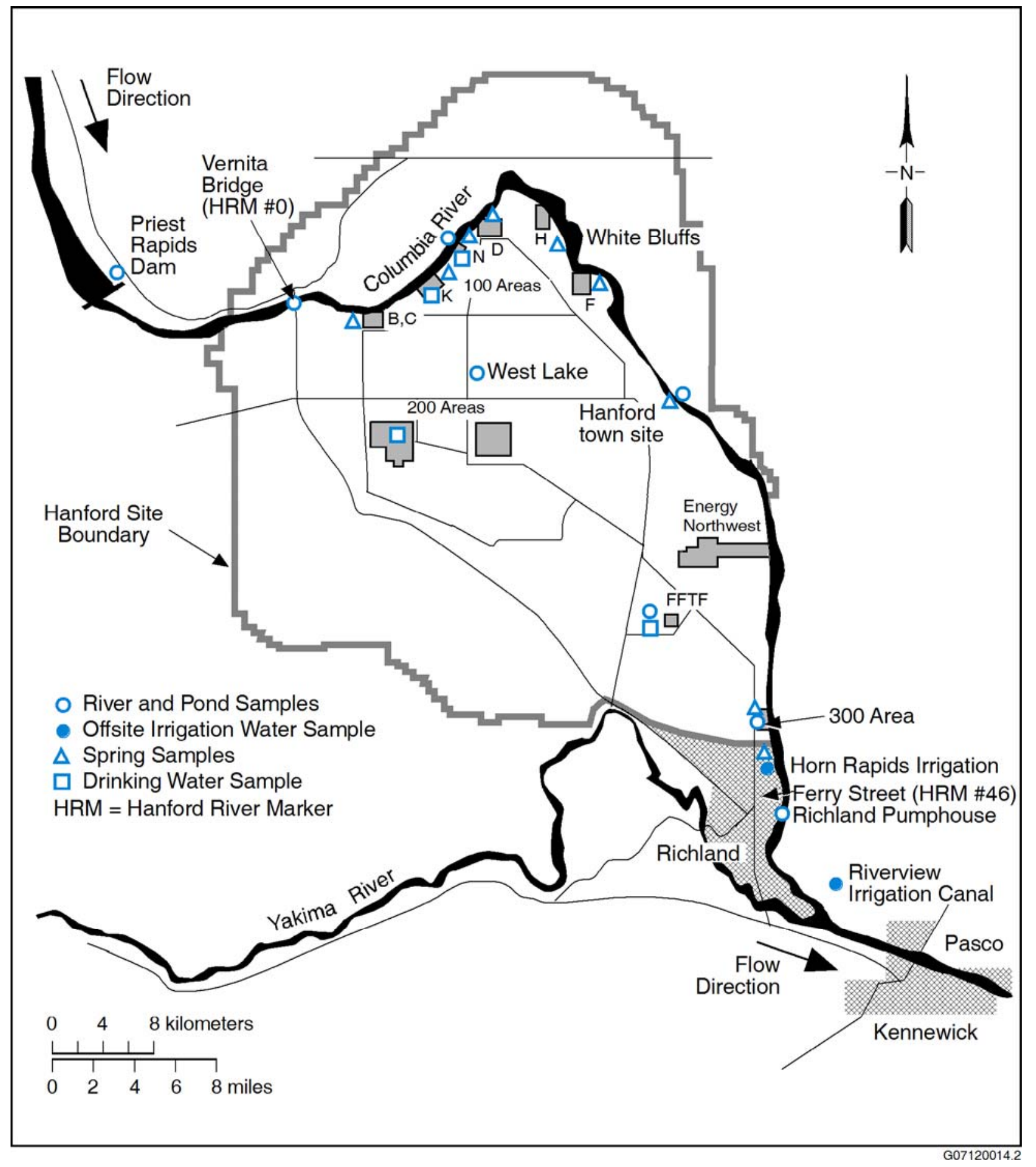

Figure 2.1. 2008 Surface Water and Drinking Water Sampling Locations 


\subsection{Drinking Water Surveillance}

\subsection{Onsite Drinking}

\begin{tabular}{|c|c|c|c|c|c|c|c|}
\hline \multirow{2}{*}{ Location $^{(a)}$} & \multirow[b]{2}{*}{$\begin{array}{c}\text { Sample } \\
\text { Type }\end{array}$} & \multicolumn{3}{|c|}{ Individual Samples } & \multicolumn{3}{|c|}{ Composited Samples } \\
\hline & & $\begin{array}{l}\text { Fre- } \\
\text { quency }\end{array}$ & Analys & ses/Agency & Composite Group & $\begin{array}{l}\text { Fre- } \\
\text { quency }\end{array}$ & Analyses/Agency \\
\hline 100 N Area & Grab & $\mathrm{M}^{(\mathrm{b})}$ & & \} & 100 N Area & $\mathrm{Q}$ & Beta \\
\hline 100 N Area & Grab & $\mathrm{Q}^{(\mathrm{c})}$ & Alpha & \} & 100 N Area & A & ${ }^{90} \mathrm{Sr},{ }^{3} \mathrm{H}$ \\
\hline 200 W Area & Grab & $\mathrm{M}^{(\mathrm{b})}$ & & \} & $200 \mathrm{~W}$ Area & $\mathrm{Q}$ & Beta \\
\hline 200 W Area & Grab & $\mathrm{Q}^{(\mathrm{c})}$ & Alpha & \} & 200 W Area & A & ${ }^{90} \mathrm{Sr},{ }^{3} \mathrm{H}$ \\
\hline $100 \mathrm{~K}$ Area & Grab & $\mathrm{M}^{(\mathrm{b})}$ & & \} & 100 K Area & $\mathrm{Q}$ & Beta \\
\hline 100 K Area & Grab & $\mathrm{Q}^{(\mathrm{c})}$ & Alpha & \} & 100 K Area & A & ${ }^{90} \mathrm{Sr},{ }^{3} \mathrm{H}$ \\
\hline 400 Area Well P-14 & Grab & $\mathrm{M}^{(\mathrm{b})}$ & & \} & 400 Area Well P-14 & Q & Beta \\
\hline 400 Area Well P-14 & Grab & $\mathrm{Q}^{(\mathrm{c})}$ & Alpha & \} & 400 Area Well P-14 & A & ${ }^{90} \mathrm{Sr},{ }^{3} \mathrm{H} / \mathrm{DOH}{ }^{(\mathrm{d})}$ \\
\hline 400 Area & Grab & $\mathrm{M}^{(\mathrm{b})}$ & & \} & 400 Area & Q & Beta \\
\hline 400 Area & Grab & $\mathrm{Q}^{(\mathrm{c})}$ & Alpha, &,$\left.{ }^{3} \mathrm{H} / \mathrm{DOH}^{(\mathrm{e})}\right\}$ & 400 Area & A & ${ }^{90} \mathrm{Sr}$ \\
\hline $\begin{array}{l}\text { (a) Refer to Figure } \\
\text { (b) Sample is collec } \\
\text { (c) Sample is collec } \\
\text { (d) Additional sam } \\
\text { (e) Additional samp }\end{array}$ & $\begin{array}{l}\text { "2008 S } \\
\text { monthly } \\
\text { and anal } \\
\text { rovided } \\
\text { rovided }\end{array}$ & $\begin{array}{l}\text { ace Wate } \\
\text { d compo } \\
\text { d quarte } \\
\text { irterly to } \\
\text { irterly to }\end{array}$ & $\begin{array}{l}\text { d Drink } \\
\text { l for qua } \\
\text { nd then } \\
\text { DOH a } \\
\text { DOH fo }\end{array}$ & $\begin{array}{l}\text { king Water Sa } \\
\text { larterly analys } \\
\text { composited } \\
\text { and then comp } \\
\text { or }{ }^{3} \mathrm{H} \text { analysis }\end{array}$ & $\begin{array}{l}\text { Locations." } \\
\text { al analysis. } \\
\text { r annual analysis. } \\
\text { y only). }\end{array}$ & & \\
\hline
\end{tabular}




\subsection{Biota}

\subsection{Food and Farm Products}

\subsubsection{Milk}

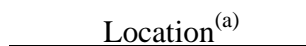

East Wahluke Area ${ }^{(b)}$

Sagemoor Composite ${ }^{(b, c)}$

Sunnyside Area
Frequency

Q

Q

Q
Analyses

Lo ${ }^{3} \mathrm{H},{ }^{90} \mathrm{Sr}$, Gamma Scan

Lo ${ }^{3} \mathrm{H},{ }^{90} \mathrm{Sr}$, Gamma Scan

Lo ${ }^{3} \mathrm{H},{ }^{90} \mathrm{Sr}$, Gamma Scan

(a) Refer to Figure 4.1, "2008 Food and Farm Products Sampling Locations."

(b) Sample composited from multiple dairies in each area.

(c) Quality assurance sample submitted for analyses once per year.

\subsubsection{Leafy Vegetables}

\section{Location $^{(\mathrm{a}, \mathrm{b})}$}

Riverview Area

Sunnyside Area

East Wahluke Area

Sagemoor Area
Frequency $^{(\mathrm{c})}$

$A$
$A$
(2008)
(2009)

Analyses/Agency

${ }^{90} \mathrm{Sr}$, Gamma Scan/FDA ${ }^{(\mathrm{d})}$

${ }^{90} \mathrm{Sr}$, Gamma Scan/FDA ${ }^{(\mathrm{d})}$

${ }^{90} \mathrm{Sr}$, Gamma Scan/DOH ${ }^{(\mathrm{e})}$

${ }^{90} \mathrm{Sr}$, Gamma Scan/DOH ${ }^{(\mathrm{e})}$

(a) Refer to Figure 4.1, "2008 Food and Farm Products Sampling Locations."

(b) Two samples collected for PNNL within each area; one sample is analyzed and one is archived.

(c) Samples are collected in 2008 according to their specified frequency unless otherwise noted.

(d) Two additional samples sent to the U.S. Food and Drug Administration (FDA).

(e) Additional sample provided to the DOH.

\subsubsection{Vegetables}

$$
\text { Location }^{(\mathrm{a}, \mathrm{b})}
$$

Riverview Area ${ }^{(\mathrm{d})}$

Sunnyside Area

East Wahluke Area

Horn Rapids Area

Sagemoor Area

$\begin{array}{cc}\text { Sample Type } & \text { Frequency }^{(\mathrm{c})} \\ \text { Potatoes } & \text { A } \\ \text { Potatoes } & \text { A } \\ \text { Potatoes } & \text { A } \\ \text { Potatoes } & \text { BE (2009) } \\ \text { Potatoes } & \text { TE (2009) }\end{array}$

Analyses/Agency

$$
\begin{aligned}
& { }^{90} \mathrm{Sr} \text {, Gamma Scan/DOH }{ }^{(\mathrm{e})} \\
& { }^{90} \mathrm{Sr} \text {, Gamma Scan/FDA }{ }^{(\mathrm{f})} \\
& { }^{90} \mathrm{Sr} \text {, Gamma Scan/DOH }{ }^{(\mathrm{e})} \\
& { }^{90} \mathrm{Sr} \text {, Gamma Scan/DOH, }{ }^{(\mathrm{e})} \mathrm{FDA}^{(\mathrm{f})} \\
& { }^{90} \mathrm{Sr} \text {, Gamma Scan/DOH, }{ }^{(\mathrm{e})} \mathrm{FDA}^{(\mathrm{f})}
\end{aligned}
$$

(a) Refer to Figure 4.1, "2008 Food and Farm Products Sampling Locations."

(b) Two samples collected for PNNL within each area; one sample is analyzed and one is archived.

(c) Samples are collected in 2008 according to their specified frequency unless otherwise noted.

(d) Other vegetables may be substituted if potatoes are not available.

(e) Additional sample provided to the DOH.

(f) Two additional samples sent to the FDA. 


\subsubsection{Fruits}

\begin{tabular}{|c|c|c|c|c|}
\hline Location $^{(\mathrm{a}, \mathrm{b})}$ & Sample Type & $\underline{\text { Frequency }}^{(\mathrm{c})}$ & $\begin{array}{c}\text { Collection } \\
\text { Period } \\
\end{array}$ & Analyses/Agency \\
\hline Sagemoor Area & $\begin{array}{l}\text { Cherries } \\
\text { Apples } \\
\text { Grapes }^{(f)}\end{array}$ & $\begin{array}{l}\text { TE (2008) } \\
\text { TE (2009) } \\
\text { TE (2010) }\end{array}$ & $\begin{array}{c}\text { June } \\
\text { September } \\
\text { September }\end{array}$ & 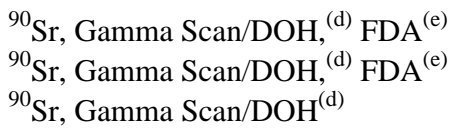 \\
\hline Sunnyside Area & $\begin{array}{l}\text { Tomatoes } \\
\text { Cherries } \\
\text { Apples } \\
\text { Grapes }^{(f)}\end{array}$ & $\begin{array}{c}\text { A } \\
\text { TE (2008) } \\
\text { TE (2009) } \\
\text { TE (2010) }\end{array}$ & $\begin{array}{l}\text { July } \\
\text { June } \\
\text { September } \\
\text { September }\end{array}$ & $\begin{array}{l}{ }^{90} \mathrm{Sr},{ }^{3} \mathrm{H}, \text { Gamma Scan } \\
{ }^{90} \mathrm{Sr}, \text { Gamma Scan } / \mathrm{DOH}^{(\mathrm{d})} \\
{ }^{90} \mathrm{Sr}, \text { Gamma Scan } / \mathrm{DOH}^{(\mathrm{d})} \\
{ }^{90} \mathrm{Sr} \text {, Gamma Scan }\end{array}$ \\
\hline Riverview Area ${ }^{(\mathrm{g})}$ & $\begin{array}{l}\text { Tomatoes } \\
\text { Cherries } \\
\text { Apples } \\
\text { Grapes }^{(f)}\end{array}$ & $\begin{array}{c}\text { A } \\
\text { TE (2008) } \\
\text { TE (2009) } \\
\text { TE (2010) }\end{array}$ & $\begin{array}{l}\text { July } \\
\text { June } \\
\text { September } \\
\text { September }\end{array}$ & $\begin{array}{l}{ }^{90} \mathrm{Sr},{ }^{3} \mathrm{H}, \text { Gamma Scan } \\
{ }^{90} \mathrm{Sr}, \text { Gamma Scan } \\
{ }^{90} \mathrm{Sr}, \text { Gamma Scan/DOH, }{ }^{(\mathrm{d})} \mathrm{FDA}^{(\mathrm{e})} \\
{ }^{90} \mathrm{Sr}, \text { Gamma Scan/DOH, }{ }^{(\mathrm{d})} \mathrm{FDA}^{(\mathrm{e})}\end{array}$ \\
\hline Ringold Area & Cherries & TE (2008) & June & ${ }^{90} \mathrm{Sr}$, Gamma Scan/DOH ${ }^{(\mathrm{d})}$ \\
\hline East Wahluke Area & Cherries & TE (2008) & June & ${ }^{90} \mathrm{Sr}$, Gamma Scan/DOH ${ }^{(\mathrm{d})}$ \\
\hline Mattawa Area & Apples & TE (2009) & September & ${ }^{90} \mathrm{Sr}$, Gamma Scan/DOH ${ }^{(\mathrm{d})}$ \\
\hline Cold Creek Area & Grapes $^{(\mathrm{f})}$ & TE (2010) & September & ${ }^{90} \mathrm{Sr}$, Gamma Scan \\
\hline
\end{tabular}

(a) Refer to Figure 4.1, “2008 Food and Farm Products Sampling Locations.”

(b) Two samples collected for the PNNL within each area; one sample is analyzed and one is archived.

(c) Samples are collected in 2008 according to their specified frequency unless otherwise noted.

(d) Additional sample provided to the $\mathrm{DOH}$.

(e) Two additional samples sent to the FDA.

(f) Concord grapes preferred; table grapes acceptable if concord grapes are unavailable.

(g) Other fruits may be substituted due to availability.

\subsubsection{Wines}

\begin{tabular}{|c|c|c|c|c|}
\hline Location $^{(\mathrm{a})}$ & $\underline{\text { Sample Type }}$ & Frequency & $\begin{array}{c}\text { Collection } \\
\text { Period } \\
\end{array}$ & Analyses/Agency \\
\hline \multirow[t]{2}{*}{ Columbia Basin $^{(b)}$} & White & BE (2009) & December & Lo ${ }^{3} \mathrm{H}$, Gamma Scan/DOH ${ }^{(\mathrm{c})}$ \\
\hline & Red & BE (2009) & December & Lo ${ }^{3} \mathrm{H}$, Gamma Scan/DOH ${ }^{(\mathrm{c})}$ \\
\hline \multirow[t]{2}{*}{ Yakima Valley } & White & BE (2009) & December & $\mathrm{Lo}^{3} \mathrm{H}$, Gamma Scan/DOH ${ }^{(\mathrm{c})}$ \\
\hline & Red & BE (2009) & December & $\mathrm{Lo}^{3} \mathrm{H}$, Gamma Scan/DOH ${ }^{(\mathrm{c})}$ \\
\hline \multirow[t]{2}{*}{ Mattawa Area } & White & BE (2009) & December & Lo ${ }^{3} \mathrm{H}$, Gamma Scan/DOH ${ }^{(\mathrm{c})}$ \\
\hline & Red & BE (2009) & December & Lo ${ }^{3} \mathrm{H}$, Gamma Scan/DOH ${ }^{(\mathrm{c})}$ \\
\hline
\end{tabular}

(a) Two samples of each type collected for PNNL within each area.

(b) Location refers to Benton and Franklin Counties.

(c) Additional sample provided to the $\mathrm{DOH}$. 


\subsubsection{Alfalfa}

\begin{tabular}{|c|c|c|c|c|}
\hline Location $^{(\mathrm{a})}$ & Sample Type & Frequency & $\begin{array}{c}\text { Collection } \\
\text { Period } \\
\end{array}$ & Analyses/Agency \\
\hline Sagemoor Area & Alfalfa & BE (2009) & May & ${ }^{90} \mathrm{Sr}$, Gamma Scan \\
\hline Riverview Area & Alfalfa & BE (2009) & May & ${ }^{90} \mathrm{Sr}$, Gamma Scan/DOH, ${ }^{(\mathrm{b})} \mathrm{FDA}^{(\mathrm{c})}$ \\
\hline Sunnyside Area & Alfalfa & BE (2009) & May & ${ }^{90} \mathrm{Sr}$, Gamma Scan/FDA ${ }^{(\mathrm{c})}$ \\
\hline Horn Rapids Area & Alfalfa & BE (2009) & May & ${ }^{90} \mathrm{Sr}$, Gamma Scan/DOH ${ }^{(\mathrm{b})}$ \\
\hline
\end{tabular}

(a) Two samples collected for PNNL within each area; one sample is analyzed and one is archived.

(b) Additional sample provided to the $\mathrm{DOH}$.

(c) Two additional samples sent to the FDA. 


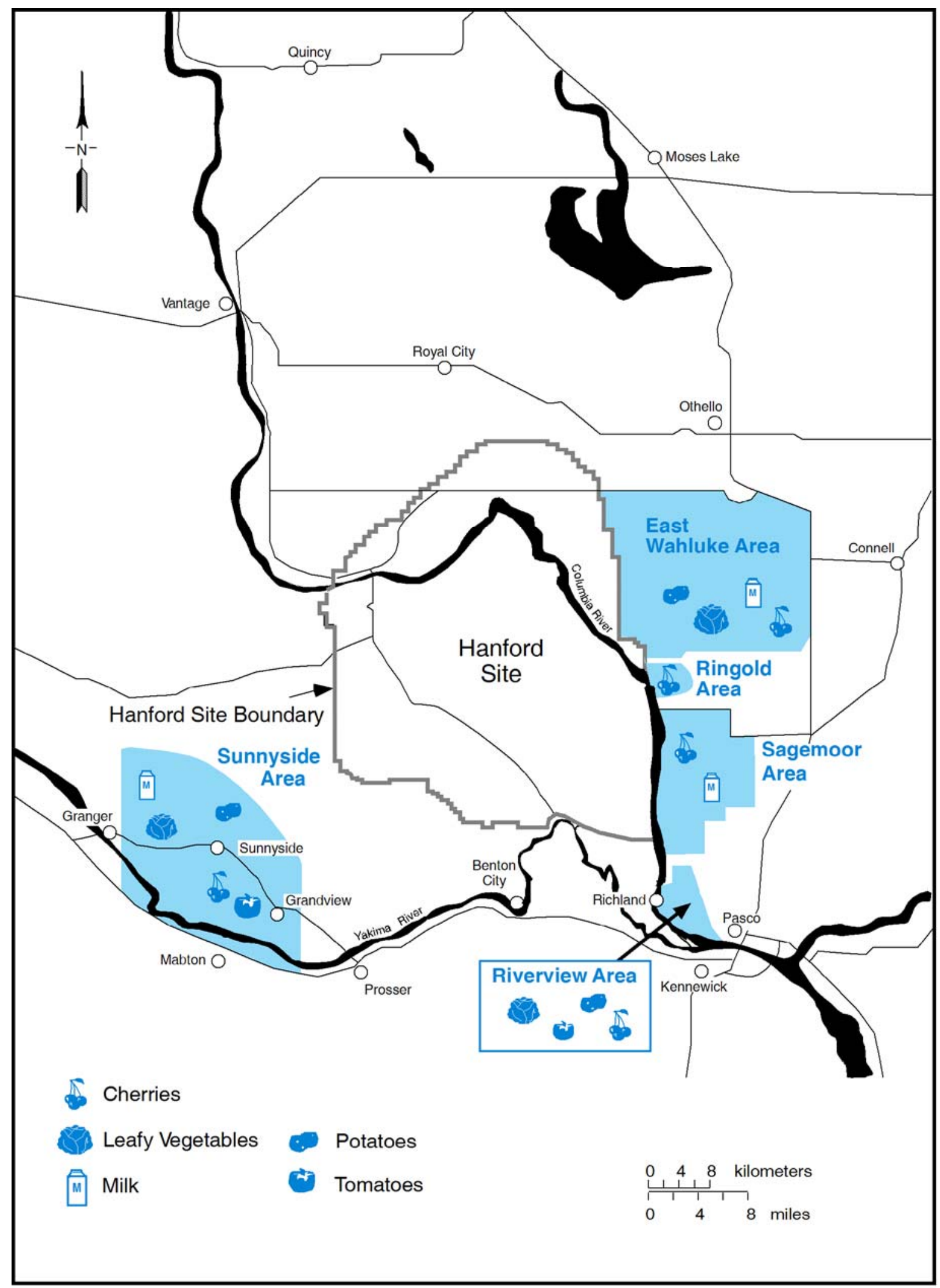

G07120014.3

Figure 4.1. 2008 Food and Farm Products Sampling Locations 


\subsection{Wildlife}

\subsubsection{Fish}

\begin{tabular}{|c|c|c|c|c|c|}
\hline Location $^{(a)}$ & $\underline{\text { Species/Sample }}$ & $\begin{array}{c}\text { Number } \\
\text { of Samples }\end{array}$ & $\underline{\text { Frequency }}^{(\mathrm{b})}$ & $\begin{array}{l}\text { Collection } \\
\text { Period }\end{array}$ & Analyses/Agency \\
\hline $100-\mathrm{N}$ to $100-\mathrm{D}$ & $\begin{array}{l}\text { Carp } \\
\text { Fillet } \\
\text { Carcass } \\
\text { Liver }^{(d)}\end{array}$ & $\begin{array}{l}5 \\
5 \\
5\end{array}$ & $\begin{array}{l}\text { BE (2008) } \\
\text { BE (2008) } \\
\text { BE (2008) }\end{array}$ & $\begin{array}{l}\text { April-July } \\
\text { April-July } \\
\text { April-July }\end{array}$ & $\begin{array}{l}\text { Gamma Scan/DOH } \\
{ }^{(\mathrm{c})} \\
{ }^{90} \mathrm{Sr} / \mathrm{DOH}^{(\mathrm{c})} \\
\text { ICP-MS }\end{array}$ \\
\hline & $\begin{array}{l}\text { Whitefish } \\
\text { Fillet } \\
\text { Carcass } \\
\text { Liver }^{(\mathrm{d})}\end{array}$ & $\begin{array}{l}5 \\
5 \\
5\end{array}$ & $\begin{array}{l}\text { BE (2009) } \\
\text { BE (2009) } \\
\text { BE (2009) }\end{array}$ & $\begin{array}{l}\text { Oct-Nov } \\
\text { Oct-Nov } \\
\text { Oct-Nov }\end{array}$ & $\begin{array}{l}\text { Gamma Scan/DOH } \\
{ }^{(\mathrm{c})} \\
{ }^{90} \mathrm{Sr} / \mathrm{DOH}^{(\mathrm{c})} \\
\text { ICP-MS }\end{array}$ \\
\hline 300 Area & $\begin{array}{l}\text { Carp } \\
\text { Fillet } \\
\text { Carcass } \\
\text { Liver }^{(d)}\end{array}$ & $\begin{array}{l}5 \\
5 \\
5\end{array}$ & $\begin{array}{l}\text { BE (2008) } \\
\text { BE (2008) } \\
\text { BE (2008) }\end{array}$ & $\begin{array}{l}\text { April-July } \\
\text { April-July } \\
\text { April-July }\end{array}$ & $\begin{array}{l}\text { Gamma Scan, U/DOH } \\
{ }^{(\mathrm{c})} \\
{ }^{90} \mathrm{Sr} / \mathrm{DOH}^{(\mathrm{c})} \\
\text { ICP-MS, Hg-CVAA }\end{array}$ \\
\hline & $\begin{array}{l}\text { Bass } \\
\quad \text { Fillet } \\
\text { Carcass } \\
\text { Liver }^{(d)}\end{array}$ & $\begin{array}{l}5 \\
5 \\
5\end{array}$ & $\begin{array}{l}\text { TE (2008) } \\
\text { TE (2008) } \\
\text { TE (2008) }\end{array}$ & $\begin{array}{l}\text { April-June } \\
\text { April-June } \\
\text { April-June }\end{array}$ & $\begin{array}{l}\text { Gamma Scan, U/DOH }^{(\mathrm{c})} \\
{ }^{90} \mathrm{Sr}_{\mathrm{DOH}}{ }^{(\mathrm{c})} \\
\text { ICP-MS, Hg-CVAA }\end{array}$ \\
\hline $\begin{array}{l}\text { Background - Desert } \\
\text { Aire/Vantage }\end{array}$ & $\begin{array}{l}\text { Carp } \\
\text { Fillet } \\
\text { Carcass } \\
\text { Liver }^{(d)}\end{array}$ & $\begin{array}{l}5 \\
5 \\
5\end{array}$ & $\begin{array}{l}\text { BE (2008) } \\
\text { BE (2008) } \\
\text { BE (2008) }\end{array}$ & $\begin{array}{l}\text { April-July } \\
\text { April-July } \\
\text { April-July }\end{array}$ & $\begin{array}{l}\text { Gamma Scan, U/DOH }^{(\mathrm{c})} \\
{ }^{90} \mathrm{Sr} \mathrm{DOH}^{(\mathrm{c})} \\
\text { ICP-MS, Hg-CVAA }\end{array}$ \\
\hline & $\begin{array}{l}\text { Bass } \\
\qquad \text { Fillet } \\
\text { Carcass } \\
\text { Liver }^{(d)}\end{array}$ & $\begin{array}{l}5 \\
5 \\
5\end{array}$ & $\begin{array}{l}\text { TE (2008) } \\
\text { TE (2008) } \\
\text { TE (2008) }\end{array}$ & $\begin{array}{l}\text { April-June } \\
\text { April-June } \\
\text { April-June }\end{array}$ & $\begin{array}{l}\text { Gamma Scan, U } \\
{ }^{90} \mathrm{Sr} \\
\text { ICP-MS, Hg-CVAA }\end{array}$ \\
\hline 100 F Slough & $\begin{array}{l}\text { Bass } \\
\quad \text { Fillet } \\
\text { Carcass } \\
\text { Liver }^{(\mathrm{d})}\end{array}$ & $\begin{array}{l}5 \\
5 \\
5\end{array}$ & $\begin{array}{l}\text { TE (2008) } \\
\text { TE (2008) } \\
\text { TE (2008) }\end{array}$ & $\begin{array}{l}\text { April-June } \\
\text { April-June } \\
\text { April-June }\end{array}$ & 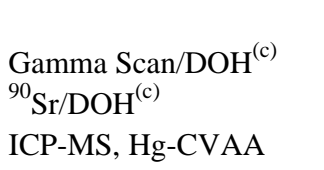 \\
\hline Hanford Slough & $\begin{array}{l}\text { Bass } \\
\text { Fillet } \\
\text { Carcass } \\
\text { Liver }^{(d)}\end{array}$ & $\begin{array}{l}5 \\
5 \\
5\end{array}$ & $\begin{array}{l}\text { TE (2008) } \\
\text { TE (2008) } \\
\text { TE (2008) }\end{array}$ & $\begin{array}{l}\text { April-June } \\
\text { April-June } \\
\text { April-June }\end{array}$ & 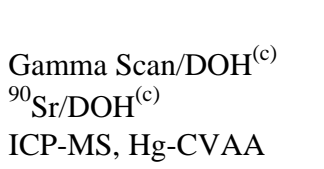 \\
\hline $\begin{array}{l}\text { Background - Priest } \\
\text { Rapids/Wanapum Pools }\end{array}$ & $\begin{array}{l}\text { Whitefish } \\
\text { Fillet } \\
\text { Carcass } \\
\text { Liver }^{(d)}\end{array}$ & $\begin{array}{l}5 \\
5 \\
5\end{array}$ & $\begin{array}{l}\text { BE (2009) } \\
\text { BE (2009) } \\
\text { BE (2009) }\end{array}$ & $\begin{array}{l}\text { November } \\
\text { November } \\
\text { November }\end{array}$ & $\begin{array}{l}\text { Gamma Scan } \\
{ }^{90} \mathrm{Sr} \\
\text { ICP-MS, Hg-CVAA }\end{array}$ \\
\hline
\end{tabular}

(a) Refer to Figure 4.2, "2008 Wildlife Sampling Locations."

(b) Samples are collected in 2008 according to their specified frequency unless otherwise noted.

(c) Additional whole fish sample provided to the $\mathrm{DOH}$.

(d) Ecological assessment sample. 


\subsubsection{Geese}

\begin{tabular}{|c|c|c|c|c|c|}
\hline Location & Species/Sample & $\begin{array}{c}\begin{array}{c}\text { Number } \\
\text { of Samples }\end{array} \\
\end{array}$ & Frequency & $\begin{array}{l}\text { Collection } \\
\text { Period } \\
\end{array}$ & Analyses \\
\hline \multirow[t]{4}{*}{100 Areas } & Canada Goose & & & & \multirow{4}{*}{$\begin{array}{l}\text { Gamma Scan } \\
{ }^{90} \mathrm{Sr} \\
\text { ICP-MS, Hg-CVAA }\end{array}$} \\
\hline & Muscle & 5 & BE (2009) & May-July & \\
\hline & Bone & 5 & BE (2009) & May-July & \\
\hline & Liver $^{(a)}$ & 5 & BE (2009) & May-July & \\
\hline \multirow{4}{*}{$\begin{array}{l}\text { Hanf Townsite to } \\
300 \text { Area }\end{array}$} & & & & & \multirow{4}{*}{$\begin{array}{l}\text { Gamma Scan } \\
{ }^{90} \mathrm{Sr} \\
\text { ICP-MS, Hg-CVAA }\end{array}$} \\
\hline & Canada Goose & & & & \\
\hline & $\begin{array}{l}\text { Muscle } \\
\text { Bone }\end{array}$ & $\begin{array}{l}5 \\
5\end{array}$ & $\begin{array}{l}\text { BE (2009) } \\
\text { BE (2009) }\end{array}$ & $\begin{array}{l}\text { May-July } \\
\text { May-July }\end{array}$ & \\
\hline & Liver $^{(a)}$ & 5 & BE (2009) & May-July & \\
\hline \multirow{4}{*}{$\begin{array}{l}\text { Background - Desert } \\
\text { Aire/Vantage }\end{array}$} & Canada Goose & & & & \\
\hline & Muscle & 5 & BE (2009) & May-July & \multirow{3}{*}{$\begin{array}{l}\text { Gamma Scan } \\
{ }^{90} \mathrm{Sr} \\
\text { ICP-MS, Hg-CVAA }\end{array}$} \\
\hline & Bone & 5 & BE (2009) & May-July & \\
\hline & Liver $^{(a)}$ & 5 & BE (2009) & May-July & \\
\hline
\end{tabular}

(a) Ecological assessment sample.

\subsubsection{Upland Game Birds}

\begin{tabular}{|c|c|c|c|c|c|}
\hline Location $^{(a)}$ & $\underline{\text { Species/Sample }}{ }^{(b)}$ & $\begin{array}{c}\begin{array}{c}\text { Number } \\
\text { of Samples }\end{array} \\
\end{array}$ & Frequency & $\begin{array}{c}\text { Collection } \\
\text { Period }\end{array}$ & Analyses/Agency \\
\hline \multirow[t]{4}{*}{$100-\mathrm{D}$ to $100-\mathrm{H}$} & Pheasant & & & & \multirow{4}{*}{$\begin{array}{l}\text { Gamma Scan } \\
{ }^{90} \mathrm{Sr} \\
\text { ICP-MS }\end{array}$} \\
\hline & Muscle & 4 & BE (2008) & September & \\
\hline & Bone & 4 & BE (2008) & September & \\
\hline & Liver $^{(\mathrm{c})}$ & 4 & BE (2008) & September & \\
\hline \multirow[t]{4}{*}{$100-\mathrm{H}$ to $100-\mathrm{F}$} & Pheasant & & & & \multirow{4}{*}{$\begin{array}{l}\mathrm{Gamma} \mathrm{Scan} \mathrm{DOH}^{(\mathrm{d})} \\
{ }^{90} \mathrm{Sr} / \mathrm{DOH}^{(\mathrm{d})} \\
\text { ICP-MS }\end{array}$} \\
\hline & Muscle & 6 & BE (2008) & September & \\
\hline & Bone & 6 & BE (2008) & September & \\
\hline & Liver $^{(\mathrm{c})}$ & 6 & BE (2008) & September & \\
\hline \multirow[t]{4}{*}{ Background } & Pheasant & & & & \\
\hline & Muscle & 5 & BE (2008) & September & \multirow{3}{*}{$\begin{array}{l}\text { Gamma Scan/DOH } \\
{ }^{(\mathrm{d})} \\
{ }^{90} \mathrm{Sr} / \mathrm{DOH}^{(\mathrm{d})} \\
\text { ICP-MS }\end{array}$} \\
\hline & Bone & 5 & BE (2008) & September & \\
\hline & Liver ${ }^{(c)}$ & 5 & BE (2008) & September & \\
\hline
\end{tabular}

(a) Refer to Figure 4.2, "2008 Wildlife Sampling Locations.”

(b) Pheasants preferred; chukars or quail acceptable if pheasants are unavailable.

(c) Ecological assessment sample.

(d) Additional whole bird sample provided to the $\mathrm{DOH}$. 


\subsubsection{Rabbits}

\begin{tabular}{|c|c|c|c|c|c|}
\hline Location & $\underline{\text { Species/Sample }}$ & $\begin{array}{c}\text { Number } \\
\text { of Samples }\end{array}$ & $\underline{\text { Frequency }}$ & $\begin{array}{c}\text { Collection } \\
\text { Period }\end{array}$ & Analyses/Agency \\
\hline \multirow[t]{4}{*}{100 N Area } & Cottontail & & & & \multirow{4}{*}{$\begin{array}{l}\text { Gamma Scan/DOH } \\
{ }^{(a)} \\
{ }^{90} \mathrm{Sr} / \mathrm{DOH}^{(\mathrm{a})} \\
\text { ICP-MS }\end{array}$} \\
\hline & Muscle & 4 & BE (2009) & Jan-Dec & \\
\hline & Bone & 4 & BE (2009) & Jan-Dec & \\
\hline & Liver $^{(b)}$ & 4 & BE (2009) & Jan-Dec & \\
\hline \multirow[t]{4}{*}{200 E Area } & Cottontail & & & & \multirow{4}{*}{$\begin{array}{l}\text { Gamma Scan/DOH } \\
{ }^{(a)} \\
{ }^{90} / \mathrm{DOH}^{(a)} \\
\mathrm{Pu}, \mathrm{ICP}-\mathrm{MS}\end{array}$} \\
\hline & Muscle & 4 & BE (2009) & Jan-Dec & \\
\hline & Bone & 4 & BE (2009) & Jan-Dec & \\
\hline & Liver $^{(b)}$ & 4 & BE (2009) & Jan-Dec & \\
\hline \multirow[t]{4}{*}{200 West } & Cottontail & & & & \multirow{4}{*}{$\begin{array}{l}\mathrm{Gamma} \mathrm{Scan} \mathrm{DOH}^{(\mathrm{a})} \\
{ }^{90} \mathrm{Sr} / \mathrm{DOH}^{(\mathrm{a})} \\
\mathrm{Pu}, \mathrm{ICP}-\mathrm{MS}\end{array}$} \\
\hline & Muscle & 4 & BE (2009) & Jan-Dec & \\
\hline & Bone & 4 & BE (2009) & Jan-Dec & \\
\hline & Liver $^{(b)}$ & 4 & BE (2009) & Jan-Dec & \\
\hline \multirow[t]{4}{*}{ Background } & Cottontail & & & & \multirow{4}{*}{$\begin{array}{l}\text { Gamma Scan } \\
{ }^{90} \mathrm{Sr} \\
\mathrm{Pu}, \mathrm{ICP}-\mathrm{MS}\end{array}$} \\
\hline & Muscle & 5 & BE (2009) & Jan-Dec & \\
\hline & Bone & 5 & BE (2009) & Jan-Dec & \\
\hline & Liver $^{(\mathrm{b})}$ & 5 & BE (2009) & Jan-Dec & \\
\hline
\end{tabular}

(a) Additional whole rabbit sample provided to the DOH.

(b) Ecological assessment sample.

\subsubsection{Deer/Elk}

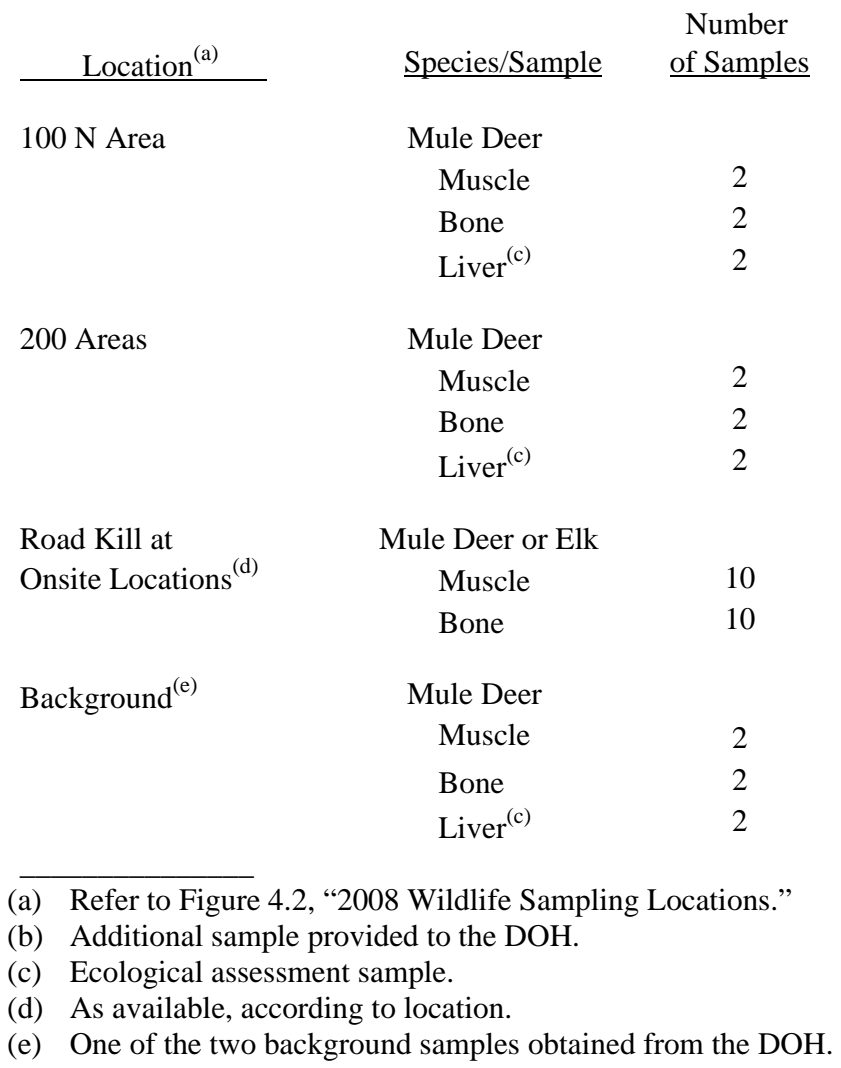

Collection

Period

BE (2008) Nov-Dec

BE (2008) Nov-Dec

BE (2008) Nov-Dec

BE (2008) Nov-Dec

BE (2008) Nov-Dec

BE (2008) Nov-Dec

BE (2008)

BE (2008)

As Available

As Available

BE (2008)
BE (2008)
BE (2008)

October

October

October
Gamma Scan

${ }^{90} \mathrm{Sr}$

Analyses/Agency

Gamma Scan/DOH ${ }^{(b)}$

${ }^{90} \mathrm{Sr} / \mathrm{DOH}^{(\mathrm{b})}$

ICP-MS

Gamma Scan/DOH ${ }^{(b)}$

${ }^{90} \mathrm{Sr} / \mathrm{DOH}^{(\mathrm{b})}$

$\mathrm{Pu}$, ICP-MS

Gamma Scan/DOH ${ }^{(b)}$

${ }^{90} \mathrm{Sr} / \mathrm{DOH}^{(\mathrm{b})}$

$\mathrm{Pu}, \mathrm{ICP}-\mathrm{MS}$ 


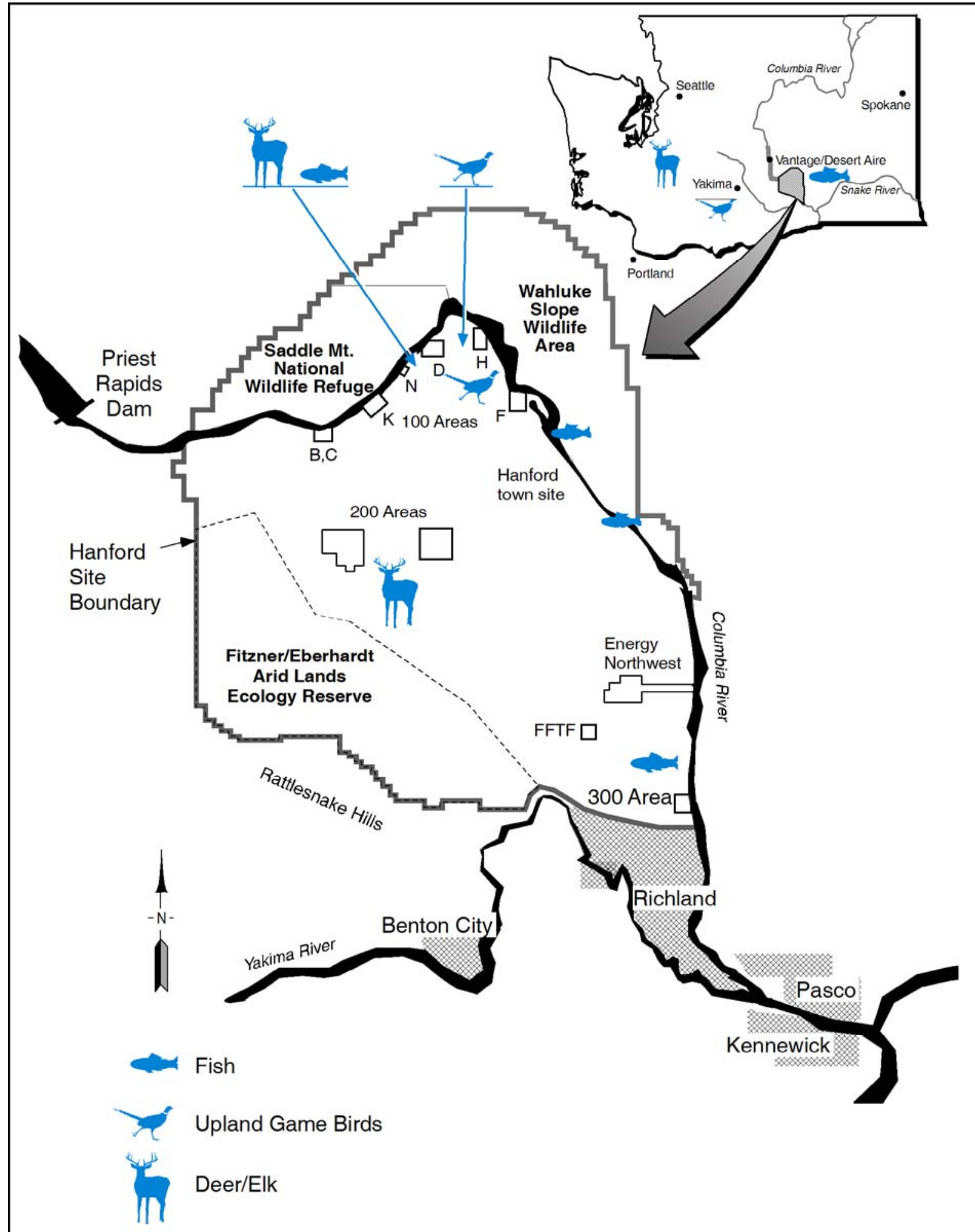

Not to Scale

G07120014.

Figure 4.2. 2008 Wildlife Sampling Locations 


\subsection{Soil and Vegetation}

\subsection{Soil}

\begin{tabular}{|c|c|c|}
\hline Location & Frequency $^{(\mathrm{a})}$ & $\begin{array}{c}\text { Collection } \\
\text { Period } \\
\end{array}$ \\
\hline $100 \mathrm{~K}$ Area & 3 to 5 yrs & June-Sept \\
\hline NE of 100 N Area & 3 to 5 yrs & June-Sept \\
\hline E of $100 \mathrm{~N}$ Area & 3 to 5 yrs & June-Sept \\
\hline 100N Shore Above HGP & 3 to 5 yrs & June-Sept \\
\hline 100N Spring Shoreline & 3 to 5 yrs & June-Sept \\
\hline Above 100D Pumphouse & 3 to $5 \mathrm{yrs}$ & June-Sept \\
\hline 100 Area Fire Stat & 3 to 5 yrs & June-Sept \\
\hline 200 ENC & 3 to 5 yrs & June-Sept \\
\hline E of $200 \mathrm{E}$ & 3 to 5 yrs & June-Sept \\
\hline 200 ESE & 3 to $5 \mathrm{yrs}$ & June-Sept \\
\hline S of $200 \mathrm{E}$ & 3 to 5 yrs & June-Sept \\
\hline SW of B/C Cribs & 3 to 5 yrs & June-Sept \\
\hline E of 200 W Gate & 3 to 5 yrs & June-Sept \\
\hline S of $200 \mathrm{~W}$ & 3 to 5 yrs & June-Sept \\
\hline Rattlesnake Springs & 3 to $5 \mathrm{yrs}$ & June-Sept \\
\hline Yakima Barricade & 3 to 5 yrs & June-Sept \\
\hline $400 \mathrm{E}$ & 3 to 5 yrs & June-Sept \\
\hline SE Side of FFTF & 3 to 5 yrs & June-Sept \\
\hline North of 300 Area & 3 to $5 \mathrm{yrs}$ & June-Sept \\
\hline South of 300 Area & 3 to 5 yrs & June-Sept \\
\hline Hanford Townsite & 3 to $5 \mathrm{yrs}$ & June-Sept \\
\hline Wye Barricade ${ }^{(\mathrm{c})}$ & 3 to $5 \mathrm{yrs}$ & June-Sept \\
\hline Prosser Barricade & 3 to $5 \mathrm{yrs}$ & June-Sept \\
\hline ALE Field Lab & 3 to 5 yrs & June-Sept \\
\hline N End Vernita Bridge & 3 to 5 yrs & June-Sept \\
\hline Wahluke Slope & 3 to 5 yrs & June-Sept \\
\hline Berg Ranch & 3 to $5 \mathrm{yrs}$ & June-Sept \\
\hline Ringold Area & 3 to 5 yrs & June-Sept \\
\hline W End of Fir Road & 3 to 5 yrs & June-Sept \\
\hline Taylor Flats No. 2 & 3 to 5 yrs & June-Sept \\
\hline Sagemoor Farm ${ }^{(\mathrm{c})}$ & 3 to 5 yrs & June-Sept \\
\hline Byers Landing & 3 to 5 yrs & June-Sept \\
\hline Riverview-Harris & 3 to 5 yrs & June-Sept \\
\hline Benton City & 3 to 5 yrs & June-Sept \\
\hline Sunnyside & 3 to 5 yrs & June-Sept \\
\hline McNary Dam & 3 to 5 yrs & June-Sept \\
\hline Walla Walla & 3 to $5 \mathrm{yrs}$ & June-Sept \\
\hline Washtucna & 3 to 5 yrs & June-Sept \\
\hline Toppenish & 3 to 5 yrs & June-Sept \\
\hline George & 3 to 5 yrs & June-Sept \\
\hline Othello & 3 to 5 yrs & June-Sept \\
\hline Wanapum & 3 to 5 yrs & June-Sept \\
\hline
\end{tabular}

\begin{tabular}{|c|}
\hline Analyses/Agency \\
\hline 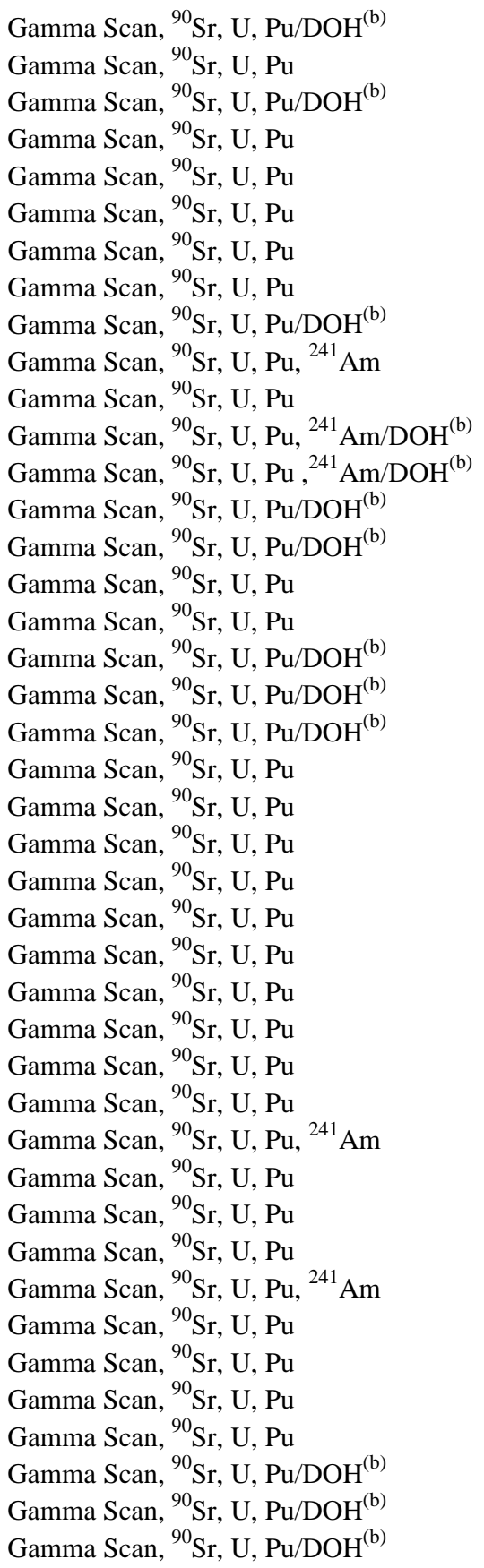 \\
\hline
\end{tabular}

ALE $=$ Fitzner/Eberhardt Arid Lands Ecology Reserve.

(a) Samples are collected once every 3 to 5 years and should be collected in 2009.

(b) Additional sample provided to the $\mathrm{DOH}$.

(c) Quality assurance samples submitted for analyses. 


\subsection{Vegetation}

\begin{tabular}{|c|c|c|c|}
\hline Location & Frequency $^{(a)}$ & $\begin{array}{c}\text { Collection } \\
\text { Period } \\
\end{array}$ & Analyses/Agency \\
\hline 100 K Area & 3 to $5 \mathrm{yrs}$ & June-Sept & Gamma Scan, ${ }^{90} \mathrm{Sr}, \mathrm{U}, \mathrm{Pu} / \mathrm{DOH}^{(\mathrm{b})}$ \\
\hline NE of $100 \mathrm{~N}$ Area & 3 to 5 yrs & June-Sept & Gamma Scan, ${ }^{90} \mathrm{Sr}, \mathrm{U}, \mathrm{Pu} / \mathrm{DOH}^{(\mathrm{b})}$ \\
\hline E of $100 \mathrm{~N}$ Area & 3 to 5 yrs & June-Sept & Gamma Scan, ${ }^{90} \mathrm{Sr}, \mathrm{U}, \mathrm{Pu}$ \\
\hline 100N Spring Shoreline & 3 to 5 yrs & June-Sept & Gamma Scan, ${ }^{90} \mathrm{Sr}, \mathrm{U}, \mathrm{Pu}$ \\
\hline E of $200 \mathrm{~W}$ Gate & 3 to 5 yrs & June-Sept & Gamma Scan, ${ }^{90} \mathrm{Sr}, \mathrm{U}, \mathrm{Pu}$ \\
\hline 300 Area Shoreline & 3 to 5 yrs & June-Sept & Gamma Scan, ${ }^{90} \mathrm{Sr}, \mathrm{U}, \mathrm{Pu} / \mathrm{DOH}^{(\mathrm{b})}$ \\
\hline Hanford Townsite & 3 to 5 yrs & June-Sept & Gamma Scan, ${ }^{90} \mathrm{Sr}, \mathrm{U}, \mathrm{Pu}$ \\
\hline Hanford Twnsite HRM28 & 3 to 5 yrs & June-Sept & Gamma Scan, ${ }^{90} \mathrm{Sr}, \mathrm{U}, \mathrm{Pu} / \mathrm{DOH}^{(\mathrm{b})}$ \\
\hline Ringold Area & 3 to 5 yrs & June-Sept & Gamma Scan, ${ }^{90} \mathrm{Sr}, \mathrm{U}, \mathrm{Pu}$ \\
\hline Sagemoor Farm & 3 to 5 yrs & June-Sept & Gamma Scan, ${ }^{90} \mathrm{Sr}, \mathrm{U}, \mathrm{Pu}$ \\
\hline Byers Landing & 3 to 5 yrs & June-Sept & Gamma Scan, ${ }^{90} \mathrm{Sr}, \mathrm{U}, \mathrm{Pu}$ \\
\hline Riverview-Harris & 3 to 5 yrs & June-Sept & Gamma Scan, ${ }^{90} \mathrm{Sr}, \mathrm{U}, \mathrm{Pu}$ \\
\hline Sunnyside & 3 to 5 yrs & June-Sept & Gamma Scan, ${ }^{90} \mathrm{Sr}, \mathrm{U}, \mathrm{Pu}$ \\
\hline Toppenish & 3 to 5 yrs & June-Sept & Gamma Scan, ${ }^{90} \mathrm{Sr}, \mathrm{U}, \mathrm{Pu}$ \\
\hline George & 3 to 5 yrs & June-Sept & Gamma Scan, ${ }^{90} \mathrm{Sr}, \mathrm{U}, \mathrm{Pu} / \mathrm{DOH}^{(\mathrm{b})}$ \\
\hline Othello & 3 to 5 yrs & June-Sept & Gamma Scan, ${ }^{90} \mathrm{Sr}, \mathrm{U}, \mathrm{Pu} / \mathrm{DOH}^{(\mathrm{b})}$ \\
\hline Wanapum & 3 to 5 yrs & June-Sept & Gamma Scan, ${ }^{90} \mathrm{Sr}, \mathrm{U}, \mathrm{Pu} / \mathrm{DOH}^{(\mathrm{b})}$ \\
\hline
\end{tabular}

(a) Samples are collected once every 3 to 5 years and should be collected in 2009 .

(b) Additional sample provided to the $\mathrm{DOH}$. 


\subsection{Sediment}

\subsection{Columbia River}

\begin{tabular}{|c|c|c|}
\hline Location $^{(a)}$ & Frequency & Analyses/Agency \\
\hline \multicolumn{3}{|l|}{ McNary Dam } \\
\hline McNary-OR. Side Near Dam & A & Gamma Scan, ${ }^{90} \mathrm{Sr}, \mathrm{U}, \mathrm{Pu}, \mathrm{ICP}-\mathrm{MS}, \mathrm{Hg}-\mathrm{CVAA}, \mathrm{TOC} / \mathrm{DOH}^{(\mathrm{b})}$ \\
\hline McNary-Wash. Side Near Dam & A & Gamma Scan, ${ }^{90} \mathrm{Sr}, \mathrm{U}, \mathrm{Pu}, \mathrm{ICP}-\mathrm{MS}, \mathrm{Hg}-\mathrm{CVAA}, \mathrm{TOC} / \mathrm{DOH}^{(\mathrm{b})}$ \\
\hline \multicolumn{3}{|l|}{ Priest Rapids Dam (PRD) } \\
\hline PRD-Grant Side Near Dam & A & Gamma Scan, ${ }^{90} \mathrm{Sr}, \mathrm{U}, \mathrm{Pu}, \mathrm{ICP}-\mathrm{MS}, \mathrm{Hg}-\mathrm{CVAA}, \mathrm{TOC} / \mathrm{DOH}^{(\mathrm{b})}$ \\
\hline PRD-Yakima Side Near Dam & A & Gamma Scan, ${ }^{90} \mathrm{Sr}, \mathrm{U}, \mathrm{Pu}, \mathrm{ICP}-\mathrm{MS}, \mathrm{Hg}-\mathrm{CVAA}, \mathrm{TOC} / \mathrm{DOH}^{(\mathrm{b})}$ \\
\hline White Bluffs Slough & A & Gamma Scan, ${ }^{90} \mathrm{Sr}, \mathrm{U}, \mathrm{Pu}, \mathrm{ICP}-\mathrm{MS}, \mathrm{Hg}-\mathrm{CVAA}$, TOC \\
\hline 100 F Slough & A & Gamma Scan, ${ }^{90} \mathrm{Sr}, \mathrm{U}, \mathrm{Pu}$, ICP-MS, Hg-CVAA, TOC \\
\hline Hanford Slough & A & Gamma Scan, ${ }^{90} \mathrm{Sr}, \mathrm{U}, \mathrm{Pu}, \mathrm{ICP}-\mathrm{MS}, \mathrm{Hg}-\mathrm{CVAA}, \mathrm{TOC} / \mathrm{DOH}^{(\mathrm{b})}$ \\
\hline Richland & A & Gamma Scan, ${ }^{90} \mathrm{Sr}, \mathrm{U}, \mathrm{Pu}, \mathrm{ICP}-\mathrm{MS}$, Hg-CVAA, TOC \\
\hline
\end{tabular}

(a) Refer to Figure 6.1, “2008 Sediment Sampling Locations.”

(b) Additional sample provided to the $\mathrm{DOH}$.

\subsection{River Shoreline Springs}

\begin{tabular}{|c|c|c|c|}
\hline Location $^{(a)}$ & $\underline{\mathrm{HRM}}^{(\mathrm{b})}$ & Frequency & Analyses/Agency \\
\hline 100-B Spring 38-3 & 3.8 & A & Gamma Scan, ${ }^{90} \mathrm{Sr}, \mathrm{U}, \mathrm{ICP}-\mathrm{MS}, \mathrm{Hg}-\mathrm{CVAA} / \mathrm{DOH}^{(\mathrm{c})}$ \\
\hline 100-K Spring 63-1 & 6.3 & A & Gamma Scan, ${ }^{90} \mathrm{Sr}, \mathrm{U}, \mathrm{ICP}-\mathrm{MS}, \mathrm{Hg}-\mathrm{CVAA}$ \\
\hline 100-H Spring 145-1 & 14.4 & A & Gamma Scan, ${ }^{90} \mathrm{Sr}, \mathrm{U}$, ICP-MS, Hg-CVAA \\
\hline 100-F Spring 207-1 & 21.3 & A & Gamma Scan, ${ }^{90} \mathrm{Sr}$, U, ICP-MS, Hg-CVAA \\
\hline Hanford Spr UR 28-2 $2^{(\mathrm{d})}$ & 27.8 & A & Gamma Scan, ${ }^{90} \mathrm{Sr}, \mathrm{U}, \mathrm{ICP}-\mathrm{MS}, \mathrm{Hg}-\mathrm{CVAA} / \mathrm{DOH}^{(\mathrm{c})}$ \\
\hline Hanford Spr DR 28-2 $2^{(\mathrm{e})}$ & 28.3 & A & Gamma Scan, ${ }^{90} \mathrm{Sr}, \mathrm{U}, \mathrm{ICP}-\mathrm{MS}, \mathrm{Hg}-\mathrm{CVAA} / \mathrm{DOH}^{(\mathrm{c})}$ \\
\hline 300 Area Spring 41-9 & 41.9 & A & Gamma Scan, U \\
\hline 300 Area Spring 42-2 & 42.1 & A & Gamma Scan, ${ }^{90} \mathrm{Sr}, \mathrm{U}, \mathrm{ICP}-\mathrm{MS}, \mathrm{Hg}-\mathrm{CVAA} / \mathrm{DOH}^{(\mathrm{c})}$ \\
\hline 300 Area Spr DR 42-2 ${ }^{(\mathrm{e})}$ & 42.4 & A & Gamma Scan, ${ }^{90} \mathrm{Sr}, \mathrm{U}, \mathrm{ICP}-\mathrm{MS}, \mathrm{Hg}-\mathrm{CVAA} / \mathrm{DOH}^{(\mathrm{c})}$ \\
\hline 300 Area Spring 42-7 & 42.7 & A & Gamma Scan, U \\
\hline Richland Spr(SRL 437-1) & 43.7 & A & Gamma Scan, U \\
\hline \multicolumn{4}{|c|}{ (a) Refer to Figure 6.1, “2008 Sediment Sampling Locations.” } \\
\hline \multicolumn{4}{|c|}{$\begin{array}{l}\text { (b) Hanford river markers (HRM) are a series of signposts along the Hanford Site shoreline of the Columbia River that are roughly } \\
1.6 \text { kilometers ( } 1 \text { mile) apart. The Vernita Bridge is HRM \#0 and Ferry Street in Richland is HRM \#46. Samples collected } \\
\text { between HRMs are assigned a decimal. }\end{array}$} \\
\hline \multicolumn{4}{|c|}{ (c) Additional sample provided to the DOH. } \\
\hline \multicolumn{4}{|c|}{ (d) UR = Upriver from noted location. } \\
\hline \multicolumn{4}{|c|}{ (e) $\mathrm{DR}=$ Downriver from noted location. } \\
\hline
\end{tabular}




\subsection{Onsite Pond}

Location $^{(\mathrm{a})}$

West Lake
Frequency

SA (Feb \& June)
Analyses/Agency

Gamma Scan, ${ }^{90} \mathrm{Sr}, \mathrm{U},{ }^{99} \mathrm{Tc}$, Alpha, Beta/DOH ${ }^{(\mathrm{b})}$

(a) Refer to Figure 6.1, "2008 Sediment Sampling Locations.”

(b) Additional sample provided to the DOH (February only).

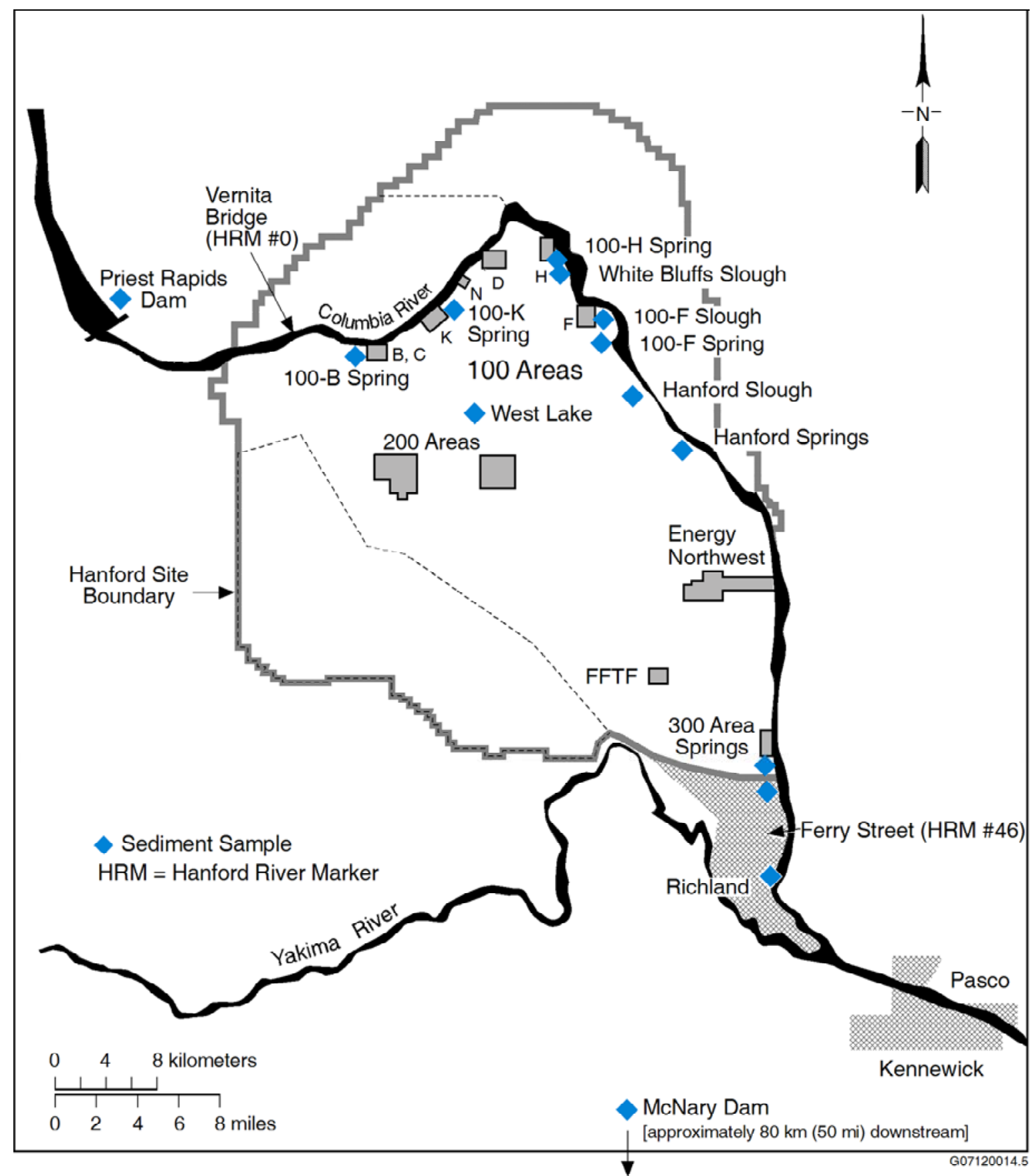

Figure 6.1. 2008 Sediment Sampling Locations 


\subsection{References}

DOE/RL-91-50, Rev. 3. 2000. Environmental Monitoring Plan, United States Department of Energy, Richland Operations Office. U.S. Department of Energy, Richland, Washington.

DOE Order 450.1. 2003. “Environmental Protection Program.” U.S. Department of Energy, Washington, D.C.

DOE Order 5400.5. 1990. "Radiation Protection of the Public and the Environment.” U.S. Department of Energy, Washington, D.C.

HEIS. 1989. Hanford Environmental Information System. Environmental Information Systems Department, Fluor Hanford, Inc., Richland, Washington 
PNNL-17282

\section{Distribution}

No. of

Copies

\section{OFFSITE (32)}

2 L. Albin

Division of Radiation Protection

Washington State Department of Health

P.O. Box 47827

Olympia, WA 98504-7827

C. Andrade

Washington Department of Fish and

Wildlife

600 Capitol Way

1111 Washington Street NE

Olympia, WA 98501-1091

G. Bohnee

Nez Perce Tribe

P.O. Box 365

Lapwai, ID 83540

R. Brunoe, GW Natural Resource

Confederated Tribes of Warm Springs

Reservation

P.O. Box 1299

Warm Springs, OR 97761

L. Buck

Wanapum

P.O. Box 878

Ephrata, WA 98823

R. Buck, Jr.

Wanapum

P.O. Box 878

Ephrata, WA 98823

L. Buelow

U.S. Environmental Protection Agency, 309 Bradley, Suite 115, MS B1-46

Richland, WA 99352

2 N. Ceto

U.S. Environmental Protection Agency

309 Bradley, Suite 115, MS B1-46

Richland, WA 99352
No. of

Copies

L. Gadbois

U.S. Environmental Protection Agency

309 Bradley, Suite 115, MS B1-46

Richland, WA 99352

J. Gear

Oregon Department of Energy

625 Marion Street NE

Salem, OR 97301-3737

L. Goldstein

Washington State Department of Ecology

P.O. Box 47600

Olympia, WA 98504

J. Hansen

U.S. Fish and Wildlife Service

11103 E. Montgomery Drive

Spokane Valley, WA 99206

B. Harper

Confederated Tribes of the Umatilla Indian

Reservation

750 Swift, Suite 14

Richland, WA 99352

S. Harris

Confederated Tribes of the Umatilla Indian

Reservation

P.O. Box 638

Pendleton, OR 97801

G. Hughes

U.S. Fish and Wildlife Service

3250 Port of Benton Boulevard

Richland, WA 99354-1670

R. Jim, Manager

Yakama Nation

2808 Main Street

Union Gap, WA 98903 
PNNL-17282

No. of

Copies

E. Kramer

Division of Radiation Protection

Washington State Department of Health

P.O. Box 47827

Olympia, WA 98504-7827

D. Landeen

Hanford Natural Resources Trustee

Council

Nez Perce Tribe

P.O. Box 365

Lapwai, ID 83540

D. McBaugh

Division of Radiation Protection

Washington State Department of Health

P.O. Box 47827

Olympia, WA 98504-7827

J. McConnaughey

Yakama Nation

P.O. Box 6066

Kennewick, WA 99336-0066

A. Nomee

Department of Natural Resources

Coeur d'Alene Tribe

P.O. Box 408

Plummer, ID 83851-9703

Office of Environmental Cleanup

Director - Hanford Project Office

$12006^{\text {th }}$ Avenue

Seattle, WA 98101

C. Pleasants

Confederated Tribes of the Colville Indian Reservation

P.O. Box 150

Nespelem, WA 99155

M. Priddy

Washington State Department of Health 309 Bradley, Suite 201

Richland, WA 99352
No. of

Copies

P. Rigdon

Yakama Nation

P.O. Box 151

Toppenish, WA 98948

M. Ritter

U.S. Fish and Wildlife Service

3250 Port of Benton Boulevard

Richland, WA 99354

B. Ruben

Washington State Department of Health

309 Bradley, Suite 201

Richland, WA 99352

P. Shaffer

Oregon Department of Energy

625 Marion Street NE

Salem, OR 97301-3737

T. Southworth

Energy Northwest

P.O. Box 968

Richland, WA 99354

S.P. Van Verst

Division of Radiation Protection

Washington State Department of Health

P.O. Box 47827

Olympia, WA 98504-7827

\section{ONSITE (69)}

\section{DOE Pacific Northwest Site Office}

J. Erickson K9-42

\section{DOE Richland Operations Office}

D.A. Brockman A7-50

B. L. Foley A6-38

J.R. Franco A3-04

A.R. Hawkins A7-50

J.P. Sands A3-04

K.M. Thompson A6-38

A.C. Tortoso A6-38

D.C. Ward (5) A3-04

J. Zeisloft A3-04

DOE Public Reading Room (2) H2-53 
No. of

Copies

\section{DOE Office of River Protection}

W. Russell

H6-60

$3 \quad$ EnergySolutions

J.J. Dorian

G1-62

S.M. McKinney

G1-62

C.J. Perkins

G1-62

4 Fluor Hanford, Inc.

D.L. Dyekman

H8-13

A.R. Johnson

H8-26

L.M. Kelly

R3-15

K.A. Peterson

H8-13

4 Washington Closure Hanford, LLC

K.A. Gano

H4-21

L.C. Hulstrom

H4-22

R.W. Ovink

H4-21

J.E. Thomson
No. of

Copies

\section{Pacific Northwest National Laboratory}

E.J. Antonio

K3-54

L.E. Bisping (20)

K6-75

J.M. Brandenberger

Sequim

R.L. Dirkes (2)

K6-75

J.L. Downs

K6-85

R.E. Durham

K6-85

J.M. Ferritto

K6-75

B.G. Fritz

K6-75

R.W. Fulton

K6-75

K. D. Hand

K6-85

B.K. Lasorsa

Sequim

E.A. Lepel

R. P. Mueller

P8-01

K6-85

B.E. Opitz

K6-75

G.W. Patton

K6-75

R.E. Peterson

K6-75

T.M. Poston

K6-75

J.A. Stegen

K3-66

Hanford Technical Library (2)

P8-55

Historical File-T.M. Poston

K6-75 